\title{
Field Effect Modulation of Electrocatalytic Hydrogen Evolution at Back-Gated Two-Dimensional MoS2 Electrodes
}

\author{
Yan Wang, Sagar Udyavara, Matthew Neurock, C. Daniel Frisbie
}

Submitted date: 19/05/2019 - Posted date: 20/05/2019

Licence: CC BY-NC-ND 4.0

Citation information: Wang, Yan; Udyavara, Sagar; Neurock, Matthew; Frisbie, C. Daniel (2019): Field Effect Modulation of Electrocatalytic Hydrogen Evolution at Back-Gated Two-Dimensional MoS2 Electrodes. ChemRxiv. Preprint.

Electrocatalytic activity for hydrogen evolution at monolayer MoS2 electrodes can be enhanced by the application of an electric field normal to the electrode plane. The electric field is produced by a gate electrode lying underneath the MoS2 and separated from it by a dielectric. Application of a voltage to the back-side gate electrode while sweeping the MoS2 electrochemical potential in a conventional manner in $0.5 \mathrm{M} \mathrm{H} 2 \mathrm{SO} 4$ results in up to a $140-\mathrm{mV}$ reduction in overpotential for hydrogen evolution at current densities of $50 \mathrm{~mA} / \mathrm{cm} 2$. Tafel analysis indicates that the exchange current density is correspondingly improved by a factor of 4 to 0.1 $\mathrm{mA} / \mathrm{cm} 2$ as gate voltage is increased. Density functional theory calculations support a mechanism in which the higher hydrogen evolution activity is caused by gate-induced electronic charge on Mo metal centers adjacent the $\mathrm{S}$ vacancies (the active sites), leading to enhanced Mo-H bond strengths. Overall, our findings indicate that the back-gated working electrode architecture is a convenient and versatile platform for investigating the connection between tunable electronic charge at active sites and overpotential for electrocatalytic processes on ultrathin electrode materials.

File list (2)

MainText_MoS2HER_051919.pdf (745.67 KiB)

view on ChemRxiv - download file

SI_MoS2HER_051819.pdf (0.90 MiB)

view on ChemRxiv - download file 


\section{Field Effect Modulation of Electrocatalytic}

\section{Hydrogen Evolution at Back-Gated Two-}

\section{Dimensional $\mathrm{MoS}_{2}$ Electrodes}

Yan Wang ${ }^{\dagger, t}$, Sagar Udyavara ${ }^{\ddagger}$, Matthew Neurock ${ }^{\ddagger}$ and, C. Daniel Frisbie $e^{\ddagger *}$

\$ Department of Chemical Engineering and Materials Science, University of Minnesota, 421

Washington Avenue SE, Minneapolis, Minnesota 55455, United States

$\dagger$ Department of Chemistry, University of Minnesota, 207 Pleasant Street SE, Minneapolis, Minnesota 55455, United States

ABSTRACT: Electrocatalytic activity for hydrogen evolution at monolayer $\mathrm{MoS}_{2}$ electrodes can be enhanced by the application of an electric field normal to the electrode plane. The electric field is produced by a gate electrode lying underneath the $\mathrm{MoS}_{2}$ and separated from it by a dielectric. Application of a voltage to the back-side gate electrode while sweeping the $\mathrm{MoS}_{2}$ electrochemical potential in a conventional manner in $0.5 \mathrm{M} \mathrm{H}_{2} \mathrm{SO}_{4}$ results in up to a $140-\mathrm{mV}$ reduction in overpotential for hydrogen evolution at current densities of $50 \mathrm{~mA} / \mathrm{cm}^{2}$. Tafel analysis indicates that the exchange current density is correspondingly improved by a factor of 4 to $0.1 \mathrm{~mA} / \mathrm{cm}^{2}$ as gate voltage is increased. Density functional theory calculations support a mechanism in which the higher hydrogen evolution activity is caused by gate-induced electronic charge on Mo metal 
centers adjacent the $\mathrm{S}$ vacancies (the active sites), leading to enhanced Mo-H bond strengths. Overall, our findings indicate that the back-gated working electrode architecture is a convenient and versatile platform for investigating the connection between tunable electronic charge at active sites and overpotential for electrocatalytic processes on ultrathin electrode materials.

Previously, we have reported field effect modulation of outer-sphere electrochemistry at two-dimensional (2D) semiconductors, ${ }^{1-3}$ such as monolayer $\mathrm{MoS}_{2}$ and 5-nm-thick $\mathrm{ZnO}$ films grown by atomic layer deposition (ALD). In these experiments, the $2 \mathrm{D}$ material is supported on a dielectric such as $\mathrm{SiO}_{2}$ with a backside gate electrode, forming a metal-oxide-semiconductor (MOS) heterostructure, as shown in Figure 1a. The backside gate may be a true metal electrode or, because of convenience, a heavily-doped Si substrate. Application of a back-gate voltage $V_{B G}$ between the gate and an electrical contact to the 2D semiconductor simultaneously shifts the conduction and valence band edges and accumulates or depletes charge carriers in the semiconductor; this is the conventional field effect employed in silicon field effect transistors (MOSFETs). ${ }^{4}$ In some sense this field effect modification of the $2 \mathrm{D}$ working electrode is akin to chemical doping in that the offset between the Fermi level $\left(E_{F}\right)$ and the band edges is controllably changed (as in n-doping or p-doping). However, the field effect is distinctly different from chemical doping in that (1) it is electronically controlled, continuously variable, and easily reversible; (2) it is the band edges that shift in response to the field, not $E_{F}$, which means that the positions of these edges with respect to redox couples in solution are changed, which in turn implies that the heterogeneous electron transfer rate constants can be tuned. 

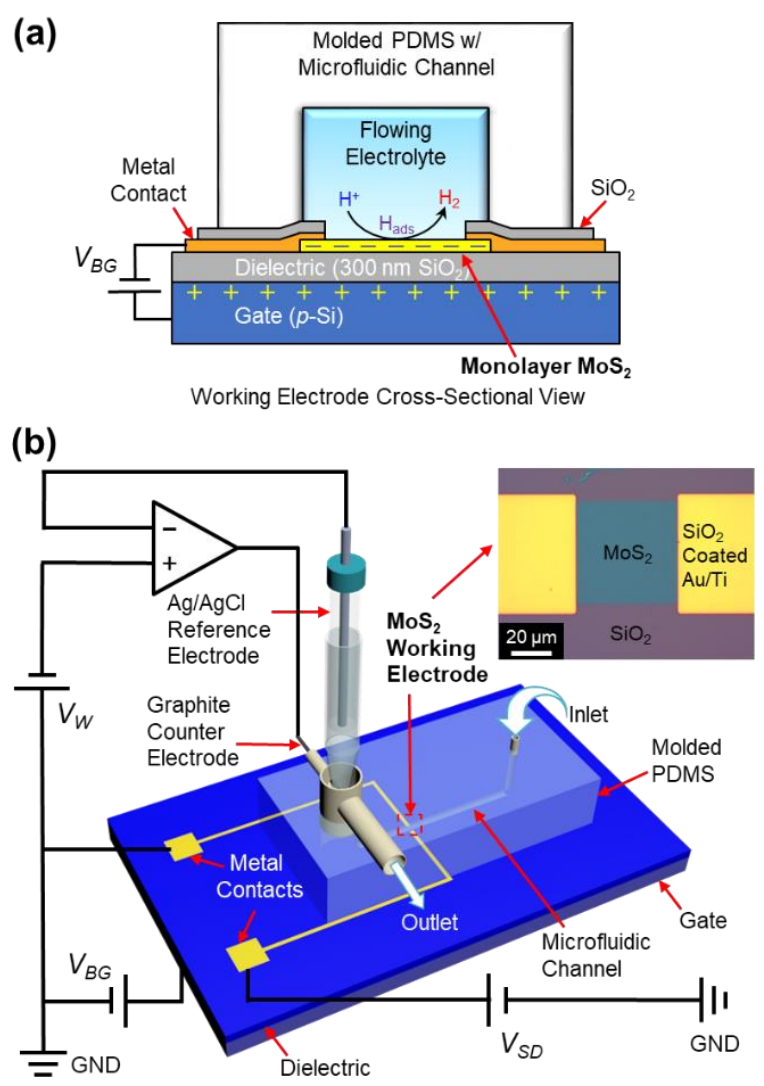

Figure 1. (a) Cross-sectional view of $\mathrm{MoS}_{2}$ working electrode and (b) 3D structure of the backgated electrochemical flow cell (not to scale) with the electrical/electrochemical configuration. The inset optical image in (b) shows a plan view of a $\mathrm{MoS}_{2}$ electrode. The symbols used in the schematics are as follows: working electrode potential $\left(V_{W}\right)$, source-to-drain bias $\left(V_{S D}\right)$, and backgate bias $\left(V_{B G}\right)$.

We have shown that field effect modulation of the band edges and carrier concentration in monolayer $\mathrm{MoS}_{2}$ and ultrathin $\mathrm{ZnO}$ can control heterogeneous charge transfer kinetics between the 2D material and simple, outer-sphere redox couples in electrolyte on the "front" side of the semiconductor. These initial experiments demonstrated the concept of field effect modulation of outer-sphere electrochemistry. ${ }^{1-3}$ Here, we take the next step and demonstrate that field effect modulation can modify the rate of an inner-sphere, electrocatalytic reaction, namely the hydrogen 
evolution reaction (HER) on $\mathrm{MoS}_{2}$. Prior reports by others have demonstrated that both polymorphs of $\mathrm{MoS}_{2}$ (metastable, metallic 1T and semiconducting 2H) are catalytically active for HER. $^{5-14}$ The activity of $2 \mathrm{H} \mathrm{MoS}$, our focus here, derives from coordinatively unsaturated Mo active sites, i.e., Mo centers adjacent $\mathrm{S}$ vacancies or at exposed crystal edges. Consequently, $\mathrm{S}$ vacancy (active site) formation is a commonly adopted strategy to activate electrocatalysis on $\mathrm{MoS}_{2},{ }^{7,15}$ though heteroatom doping is also employed. ${ }^{16-18}$

Prior to this work, the field effect has been reported to improve HER activity at back-gated multilayer $\mathrm{MoS}_{2}$ electrodes. ${ }^{19}$ However, in that work (see also a related paper on gated multilayer $\mathrm{VSe}_{2}{ }^{20}$ ), the enhanced HER activity was attributed to increased conductivity of the multilayer $\mathrm{MoS}_{2}$ (20-30 nm thick) upon gating and not to any changes in the intrinsic reactivity of the active sites. Herein, we demonstrate by a combination of experiment and density functional theory (DFT) calculations that field effect enhancement of HER activity on monolayer $\mathrm{MoS}_{2}$ occurs by a different mechanism, namely the gate-induced charging of the localized Mo d-states at the active (S-vacancy) sites, and the resultant increase of the Mo-H binding energy. In this mechanism, the increase in HER activity is due to an increase in the intrinsic reactivity of the active sites in monolayer $\mathrm{MoS}_{2}$ on applying a positive gate bias $V_{B G}$. Experimentally, we achieve a $140-\mathrm{mV}$ decrease in $\eta$ at $50 \mathrm{~mA} / \mathrm{cm}^{2}$ and a 4-fold increase in the exchange current density $i_{0}$ by varying $V_{B G}$. We systematically eliminate the possibility that the enhancement in $i_{0}$ and the decrease in $\eta$ are due to changes in the $\mathrm{MoS}_{2}$ conductivity or the resistance of the electrical contacts to $\mathrm{MoS}_{2}$. Further, using DFT, we demonstrate that the charging of the Mo centers at the active sites leads to a $140 \%$ increase in the Mo-H bond energy, which provides a clear explanation for the enhanced HER activity. We believe these experiments and associated theoretical calculations open up new 
possibilities for investigating and understanding electrocatalysis at 2D materials, as we describe below.

Monolayer $\mathrm{MoS}_{2}$ was synthesized by chemical vapor deposition (CVD) at $850^{\circ} \mathrm{C}$ following a modification of previously reported methods. ${ }^{21,22}$ Large monolayer flakes that are desirable for back-gated electrocatalysis experiments were characterized by atomic force microscopy (AFM), Raman spectroscopy, and photoluminescence and confirmed the $2 \mathrm{H}$ phase (Figure S2). After the transfer of a monolayer of $\mathrm{MoS}_{2}$ onto $\mathrm{SiO}_{2} / \mathrm{p}-\mathrm{Si}$ back gates, fabrication of $\mathrm{MoS}_{2}$ electrodes was accomplished in a series of steps including photolithography, plasma etching, e-beam evaporation of Ti/Au metal contacts, and $\mathrm{SiO}_{2}$ passivation (see the Supporting Information for details of synthesis and device fabrication). The two Ti/Au contacts (source and drain) were made to the front-side of $\mathrm{MoS}_{2}$ and the completed structure in Figure 1a can thus be viewed as a FET. From the $\mathrm{MoS}_{2}$ sheet conductance-gate voltage $\left(G_{S}-V_{B G}\right)$ characteristics, Figure S5, we confirmed the n-type behavior of $\mathrm{MoS}_{2}$ with an on/off current ratio of $\sim 10^{4}$ and a FET electron mobility of $\sim 22 \mathrm{~cm}^{2} \mathrm{~V}^{-1} \mathrm{~s}^{-1}$, which further confirmed that the monolayer $\mathrm{MoS}_{2}$ was the $2 \mathrm{H}$ phase.

To perform electrochemical measurements, a molded polydimethylsiloxane (PDMS) block with an embedded $200-\mu \mathrm{m}$ wide microfluidic channel was assembled onto a $\mathrm{MoS}_{2}$ electrode, Figure 1b. Integration of this flow cell structure facilitated forced convection of electrolyte, thereby minimizing mass transport effects on voltammetry and helping to sweep away gas bubbles generated on the $\mathrm{MoS}_{2}$ working electrode. Figure 2a displays our principal results, namely the polarization curves in flowing $0.5 \mathrm{M} \mathrm{H}_{2} \mathrm{SO}_{4}$ upon sweeping the $\mathrm{MoS}_{2}$ working electrode potential $V_{W}$ while applying a constant $V_{B G}$. At $V_{B G}=0 \mathrm{~V}$, the HER overpotentials $\left(\eta=\left|V_{w}-U^{0}\right|\right)$ at current densities of 10 and $50 \mathrm{~mA} / \mathrm{cm}^{2}$ were 294 and $474 \mathrm{mV}$, respectively. These $\eta$ values at 
$V_{B G}=0 \mathrm{~V}$ are comparable to those previously reported for HER on monolayer $\mathrm{MoS}_{2 .}{ }^{7,15,23,24}$ As $V_{B G}$ increased from $0 \mathrm{~V}$ to $+100 \mathrm{~V}$ at $20 \mathrm{~V}$ intervals, $\eta$ progressively decreased to $184 \mathrm{mV}$ (at 10 $\mathrm{mA} / \mathrm{cm}^{2}$ ) and $334 \mathrm{mV}$ (at $50 \mathrm{~mA} / \mathrm{cm}^{2}$ ), respectively (Table 1), indicating an overall improved catalytic activity for HER. Negative $V_{B G}$, on the other hand, suppressed HER on back-gated $\mathrm{MoS}_{2}$, resulting in an increased $\eta$. The effect of $V_{B G}$ on HER activity was reversible, i.e., switching of $V_{B G}$ to higher and lower values produced corresponding voltammograms that retraced themselves. As a control, we tested back-gated working electrodes in which $\mathrm{MoS}_{2}$ was replaced with graphene, Figure 2a. We observed no significant HER activity for back-gated graphene at any value of $V_{B G}$ (Figure S7), consistent with expectations. We also verified that the observed shift in $\eta$ was not a result of the ohmic drop across the $\mathrm{MoS}_{2}$ working electrode. To check that, we measured the sheet resistance of $\mathrm{MoS}_{2}$ electrodes simultaneously during the electrochemical measurement (Figure S6a). The calculated in-plane polarization at varying $V_{B G}$ and $V_{W}$ was always smaller than $11 \mathrm{mV}$ (Figure S6b) and accounted for less than $1 \%$ of the overall polarization (i.e. $\eta$ ) at current densities of 10 and $50 \mathrm{~mA} / \mathrm{cm}^{2}$ (Figure S6c). Therefore, it is clear that the reduced $\eta$ did not result from changes in the in-plane ohmic losses. 

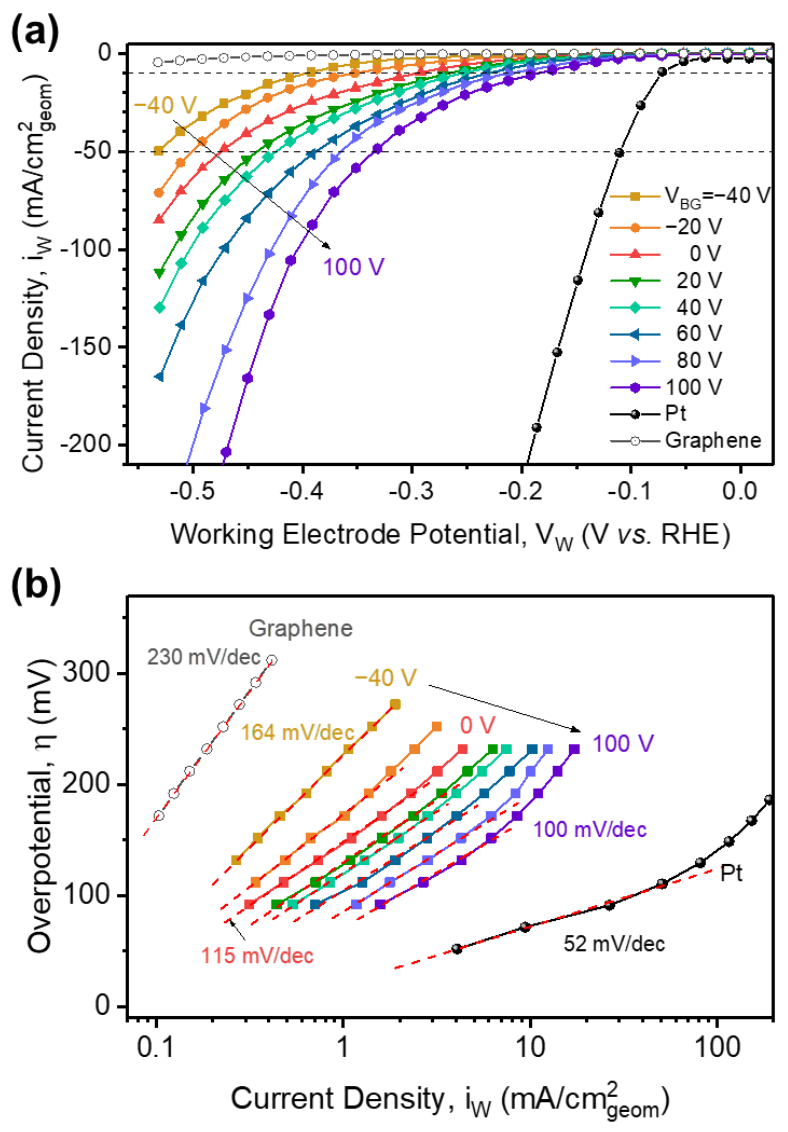

Figure 2. (a) Polarization curves and (b) corresponding Tafel plots of $0.5 \mathrm{M} \mathrm{H}_{2} \mathrm{SO}_{4}$ solution on a back-gated $\mathrm{MoS}_{2}$ electrode at different back-gate biases $V_{B G}$. Results on Pt and graphene electrodes are included for comparison. The polarization curves are iR-corrected for solution resistance. All the electrochemical measurements were performed at a working electrode potential scan rate of 50 $\mathrm{mV} / \mathrm{s}$ and an electrolyte volumetric flow rate of $10 \mu \mathrm{L} / \mathrm{min}$. 
Table 1. Overpotential, Tafel Slope, and Exchange Current Density at Varying Back-Gate Biases

\begin{tabular}{lllll}
\hline $\begin{array}{l}\boldsymbol{V}_{\boldsymbol{B}} \\
(\mathbf{V})\end{array}$ & $\begin{array}{l}\boldsymbol{\eta} \text { at 10 } \\
\mathbf{m A} / \mathbf{c m}^{\mathbf{2}}(\mathbf{m V})\end{array}$ & $\begin{array}{l}\boldsymbol{\eta} \text { at 50 } \\
\mathbf{m A} / \mathbf{c m}^{\mathbf{2}}(\mathbf{m V})\end{array}$ & $\begin{array}{l}\text { Tafel Slope } \\
(\mathbf{m v} / \mathbf{d e c})\end{array}$ & $\begin{array}{l}\boldsymbol{i}_{\mathbf{0}} \\
\left(\mathbf{m A} / \mathbf{c m}^{2}\right)\end{array}$ \\
\hline $\mathbf{- 4 0}$ & 396 & 532 & 164 & 0.030 \\
$\mathbf{- 2 0}$ & 352 & 502 & 130 & 0.032 \\
$\mathbf{0}$ & 294 & 474 & 115 & 0.034 \\
$\mathbf{2 0}$ & 266 & 442 & 113 & 0.047 \\
$\mathbf{4 0}$ & 254 & 424 & 111 & 0.054 \\
$\mathbf{6 0}$ & 230 & 388 & 106 & 0.067 \\
$\mathbf{8 0}$ & 211 & 365 & 105 & 0.098 \\
$\mathbf{1 0 0}$ & 184 & 334 & 100 & 0.122 \\
\hline
\end{tabular}

The polarization curves in Figure 2a were converted into corresponding Tafel plots, Figure $2 \mathrm{~b}$, to assess the reaction mechanism. Tafel slopes $(b)$ and exchange current densities $\left(i_{0}\right)$ were obtained by fitting the plots to $\eta=b\left(\log \left(i_{w}\right)-\log \left(i_{0}\right)\right)$. As expected, a Tafel slope of 115 $\mathrm{mV} /$ decade is observed for HER on $\mathrm{MoS}_{2}$ at $V_{B G}=0 \mathrm{~V}$, consistent with $\sim 120 \mathrm{mV} /$ decade that others have reported for monolayer $\mathrm{MoS}_{2}$ on $\mathrm{Si}_{\mathrm{SiO}}{ }_{2}{ }^{9}$ The Tafel slope gradually decreases to 100 $\mathrm{mV} /$ decade with increasing $V_{B G}$, while it increases to $164 \mathrm{mV} /$ decade at negative $V_{B G}$. It is widely accepted that HER on $\mathrm{MoS}_{2}$ follows the Volmer-Heyrovsky mechanism, where $H^{+}+e^{-} \rightleftharpoons H_{\text {ads }}$ (Volmer) and $H^{+}+e^{-}+H_{a d s} \rightleftharpoons H_{2}$ (Heyrovsky) are the elementary steps. ${ }^{25}$ Tafel slopes of $\sim 120$ $\mathrm{mV} /$ decade or larger suggest that the Volmer reaction, namely the coupled adsorption and charge transfer step, limits the overall reaction rate. ${ }^{26}$ The slight decrease in the Tafel slope indicates that there is likely not a significant change in the reaction mechanism upon varying $V_{B G}$. However, the exchange density $i_{0}$ increases by a factor of 4 from 0.034 to $0.122 \mathrm{~mA} / \mathrm{cm}^{2}$ as $V_{B G}$ increases to $+100 \mathrm{~V}$, Table 1 and Figure 3. These $i_{0}$ values obtained on back-gated $\mathrm{MoS}_{2}$ are among the highest 
reported to date, ${ }^{7-9,11}$ and the considerable increase in $i_{0}$ suggests substantially enhanced catalytic activity at equilibrium.

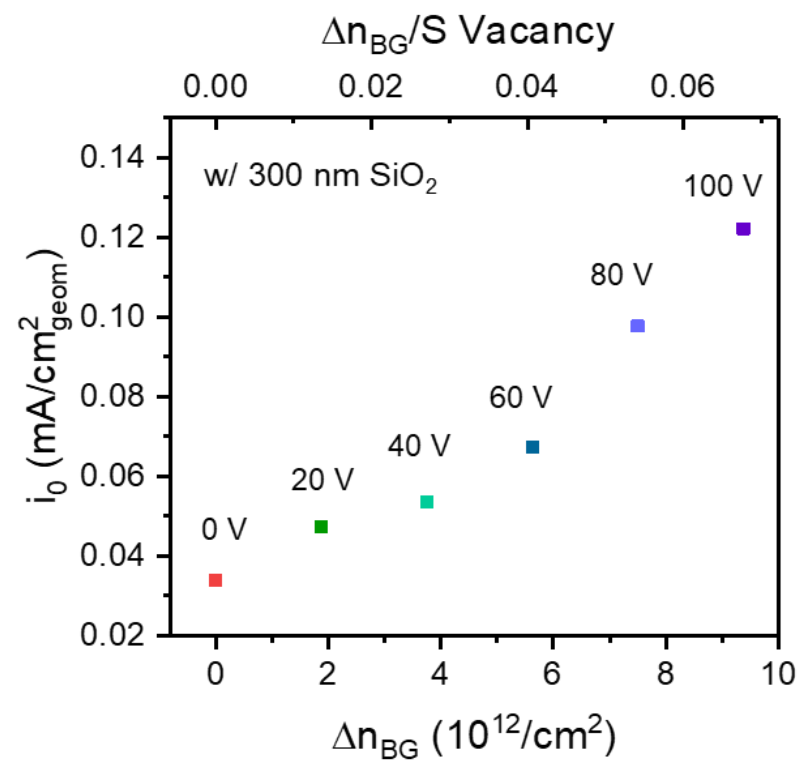

Figure 3. Exchange current density $i_{0}$ as a function of the back-gate induced charge carrier density, $\Delta n_{B G}$. The $\mathrm{x}$-axis is quoted in electrons $/ \mathrm{cm}^{2}$ and electrons $/ \mathrm{S}$ vacancy.

With more than a $100 \mathrm{mV}$ shift in $\eta$ at $10 \mathrm{~mA} / \mathrm{cm}^{2}$ and a 4-fold increase in $i_{0}$, it is reasonable to conclude that the overall $\mathrm{HER}$ activity of monolayer $2 \mathrm{H} \mathrm{MoS}_{2}$ is improved by the field effect. In order to clarify the mechanism for enhanced activity, DFT calculations were performed. The Mo centers adjacent to $\mathrm{S}$ vacancies on the $\mathrm{MoS}_{2}$ basal plane are considered to be the active sites for HER. ${ }^{7}$ X-ray photoelectron spectroscopy (XPS) analysis of the CVD monolayer $\mathrm{MoS}_{2}$ used in this study indicate that there are $\sim 6 \% \mathrm{~S}$ vacancies (Figure S3) that function as active sites. We therefore constructed a monolayer $\mathrm{MoS}_{2}$ surface comprised of 5.5\% $\mathrm{S}$ vacancies to serve as a model for DFT calculations, as shown in Figure 4a. The creation of $\mathrm{S}$ vacancies within the $\mathrm{MoS}_{2}$ surface resulted in the formation of mid-gap electronic states (see Figure 4b and S9) 
comprised predominantly of localized Mo d-states that favorably bind protons. ${ }^{7}$ To model the electrostatic doping effect of the back-gate bias on these mid-gap states, we charged the model substrate with extra electrons corresponding to the excess electron densities obtained experimentally (see Supporting Information for further details on the computational methods used). Excess electron densities of nearly $1 \times 10^{13} \mathrm{~cm}^{-2}$ as calculated by $\Delta n_{B G}=C_{S_{i O}} \times V_{B G}$ ( $C_{\mathrm{SiO}_{2}}$ is the specific capacitance of $300 \mathrm{~nm} \mathrm{SiO}$, measured to be $\sim 15 \mathrm{nF} / \mathrm{cm}^{2}$, Figure S8), are induced experimentally at $V_{B G}=+100 \mathrm{~V}$. Figure 4c displays the DFT-calculated electron density difference map corresponding to the difference in charge density at $V_{B G}=+100 \mathrm{~V}\left(\Delta n_{B G}=10^{13}\right.$ $\left.\mathrm{cm}^{-2}\right)$ versus that at $0 \mathrm{~V}\left(\Delta n_{B G}=0\right)$. From this figure, it is evident that the excess electron density induced upon the application of $V_{B G}$ is concentrated on the Mo atoms near the $\mathrm{S}$ vacancy site. This accumulation of excess electron density on the Mo centers in turn leads to a downward shift of the mid-gap states closer to $E_{F}$ as $V_{B G}$ is increased from 0 to $+100 \mathrm{~V}$ as shown in Figure $4 \mathrm{~b}$. This downward shift of the energy levels is in agreement with expectations based on the known operating principles for field effect devices. 

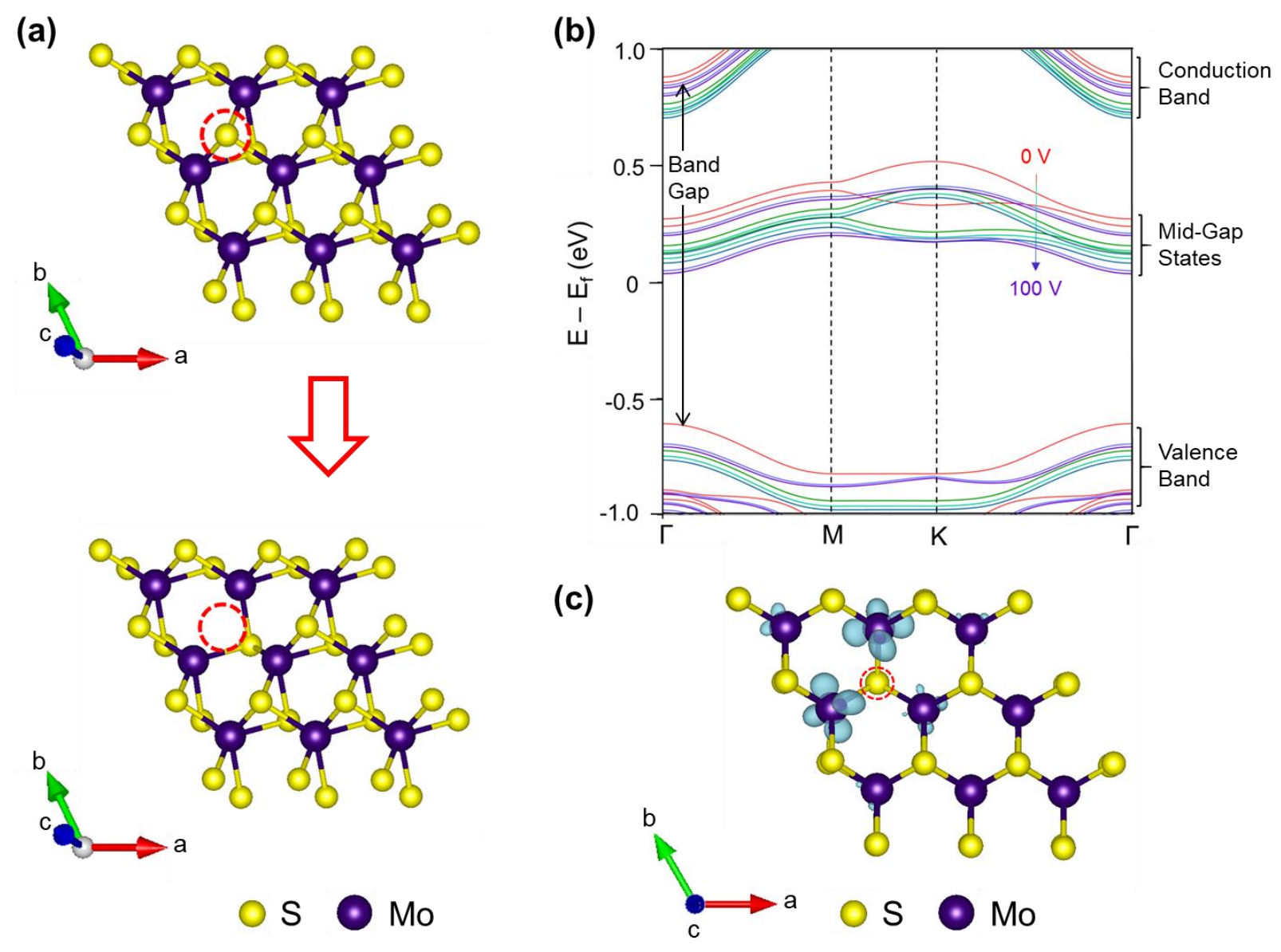

(c)

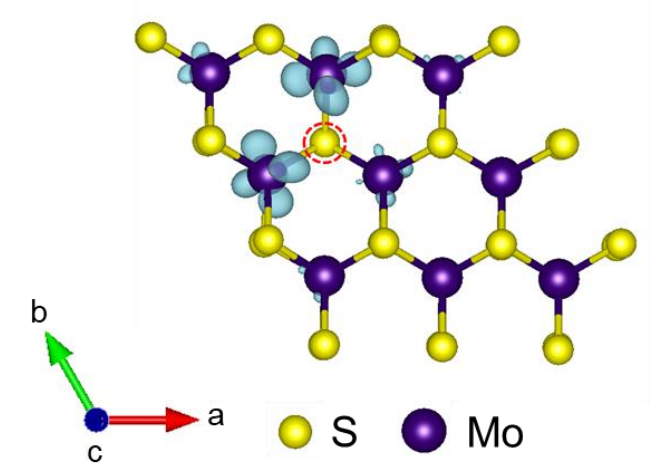

Figure 4. (a) Schematic of the S vacancy site (denoted by red dotted circle) created on model $\mathrm{MoS}_{2}$ surface used for DFT calculations (b) Computed band structures of $\mathrm{MoS}_{2}$ surface with 5.5 $\% \mathrm{~S}$ vacancy at different induced charges varying from $\Delta n_{B G}=0\left(\mathrm{~V}_{B G}=0 \mathrm{~V}\right)$ to $\Delta n_{B G}=$ $10^{13} \mathrm{~cm}^{-2}\left(V_{B G}=100 \mathrm{~V}\right)$. The band structure at $\mathrm{V}_{B G}=0 \mathrm{~V}$ is shown in red. (c) Charge density difference map (isosurface value $=0.0002 \mathrm{e}-/ \mathrm{Bohr}^{3}$ ) of monolayer $\mathrm{MoS}_{2}$ with $5.5 \% \mathrm{~S}$ vacancy showing the positive difference in electron density at an induced charge carrier density of $10^{13}$ $\mathrm{cm}^{-2}$ versus that at no excess charge density. The maps show localization of excess charge on the Mo atoms near the sulfur vacancy denoted by red circle.

The binding energy of atomic hydrogen $\left(\Delta E_{H}\right)$ to an electrode surface has been shown to be a good descriptor for trends in HER activity. ${ }^{5}$ Thus, we calculated $\Delta E_{H}$ for the binding of 
hydrogen to the $\mathrm{S}$ vacancy site at charge densities corresponding to the applied back-gate voltages. As shown in Figure $5 \mathrm{a}, \Delta E_{H}$ becomes significantly more exothermic at higher $\Delta n_{B G}$. Importantly, this stronger $\mathrm{H}$ binding provides a clear rationale for higher activity of adsorption-limited HER on $M_{o} S_{2}$; increased $\Delta E_{H}$ lowers the activation energy for the Volmer step and leads to a higher fraction of hydrogenated active sites, i.e., equilibrium is shifted in favor of $\mathrm{H}_{\text {ads }}$.

In keeping with this analysis, we observed an exponential dependence of the experimental $i_{0}$ on the calculated $\Delta E_{H}$ values as shown in Figure $5 \mathrm{~b}$. This exponential increase is expected as $i_{0}$ is an exponential function of the activation barrier for the rate-limiting Volmer step. ${ }^{27,28}$ The Figure $5 \mathrm{~b}$ trend is also consistent with previous reports that show $i_{0}$ increases exponentially with $\Delta E_{H}$ for electrocatalysts that lie on the right side of the $i_{0} v s . \Delta E_{H}$ "volcano plot", that is those that bind hydrogen weakly $\left(\Delta E_{H}>-0.2 \mathrm{eV}\right) .{ }^{5,28-30}$ Thus, the detailed DFT calculations reported here strongly support a mechanism in which the gate-induced charge enhances the intrinsic activity of the $\mathrm{MoS}_{2}$ active sites, in particular by stabilizing $\mathrm{H}_{\text {ads }}$. 

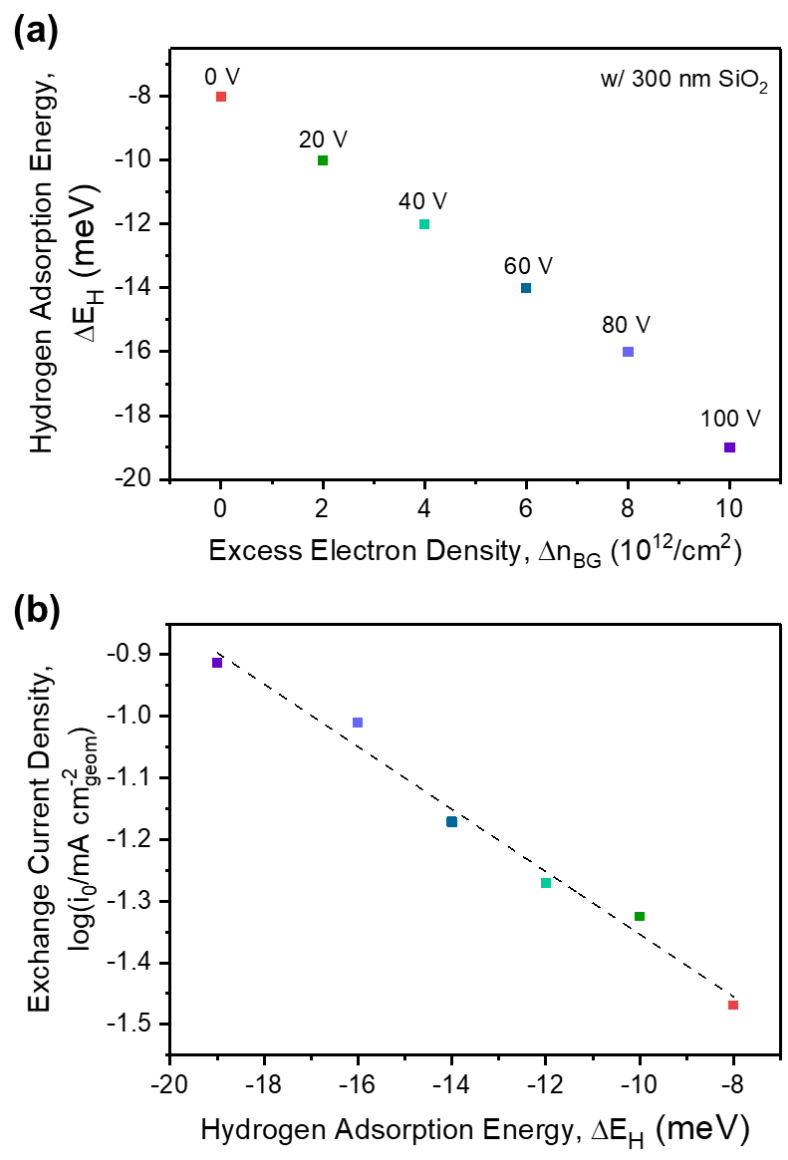

Figure 5. (a) Hydrogen adsorption energies $\left(\Delta E_{H}\right)$ as function of induced excess electron density $\left(\Delta n_{B G}\right.$ ) for values ranging from 0 to $10^{13} \mathrm{~cm}^{-2}$ (b) Experimentally determined exchange current densities $i_{0}$ for hydrogen evolution on back-gated monolayer $\mathrm{MoS}_{2}$ versus calculated $\Delta E_{H}$.

The results reported here are distinctly different than previous work reported on field effect modulation of HER at multilayer (30-nm thick) $\mathrm{MoS}_{2}$, which indicate that the decrease in $\eta$ with applied gate voltage is the result of conductance changes across the $\mathrm{MoS}_{2}$ flake, i.e., to changes in the ohmic transport of charge from the contacts to the catalytic interface. ${ }^{19}$ As mentioned above, using methods that we have previously reported ${ }^{1}$ we categorically rule out the possibility that the changes in $\eta$ are due to in-plane transport effects or to contact resistance. ${ }^{9,19}$ The changes in $\eta$ that we observe are instead due to changes in inherent catalytic activity of monolayer $\mathrm{MoS}_{2}$. This is a 
distinctly different mechanism from that proposed previously. Moreover, it does not seem likely that appreciable gate-induced enhancement of S-vacancy activity could be observed in $25-30 \mathrm{~nm}$ thick multilayer $\mathrm{MoS}_{2}$ due to electrostatic confinement and screening of the gate-induced charge at the $\mathrm{MoS}_{2}$ /gate dielectric interface. For example, we estimate the Thomas-Fermi screening length in $\mathrm{MoS}_{2}$ at $\Delta n_{B G}=10^{13} \mathrm{~cm}^{-2}$ to be approximately 1-3 nm, smaller than the $25-30 \mathrm{~nm}$ thickness of multilayer $\mathrm{MoS}_{2}$.

To our knowledge this is the first report of field-effect modulation to change the intrinsic activity of electrocatalytic sites on ultrathin, back-gated electrodes. To be clear, by "field-effect modulation" we mean the back-gating method we have employed here, not the normal doublelayer charging that occurs in conventional electrochemical experiments. It is important to note that the back gate provides a degree of freedom that is not generally available in conventional electrocatalysis experiments. In a conventional electrocatalysis experiment, double-layer charging of active sites surely occurs but there is no ability to reversibly change the degree of charging independent of the electrochemical potential. With the back gate on sufficiently thin electrocatalyst layers, the amount of charge in the active sites is independently controlled and the impact on the overpotential can then be assessed directly and immediately, as demonstrated here.

There are other reports of electronic modification of gas-phase heterogeneous catalysts that have intriguing analogies to our experiments. ${ }^{31,32}$ An example is the use of voltage-controlled Schottky junctions to manipulate electronic occupation in thin catalytic layers grown on doped semiconductors. ${ }^{32}$ However, the use of an intervening dielectric in the platform we describe here can dramatically boost accumulated charge concentrations and provides flexibility in $2 \mathrm{D}$ electrode design as the alignment of the electrode band edges with the substrate is not a primary consideration. 
The back-gated platform described here offers ample opportunities for further investigations of electrocatalysis at 2D electrodes for several reasons. First, the advent of ALD and 2D materials synthesis provides a rich palette of ultra-thin materials that can be coupled to gate/dielectric stacks and implemented as back-gated working electrodes. Second, the continuous, reversible electrostatic doping that is achieved in this platform can be a powerful complement to chemical (e.g., heteroatom) doping strategies for optimizing 2D electrocatalysts. It will be interesting to understand in what ways electrostatic doping and chemical doping are similar or dissimilar. Third, as noted above, the independent control of electrode charging afforded by the back gate portends important opportunities to understand the differences in active site promotion by field-effect versus double-layer charging.

We note that the electrode architecture we have presented here is not fully optimized. For example, switching to high dielectric constant insulators, such as $\mathrm{HfO}_{2}$, offers the possibility of simultaneously decreasing the required gate voltages and increasing the gate-induced carrier densities up to $10^{14} \mathrm{~cm}^{-2}$. Preliminary computational results suggest a nearly 10 -fold stronger hydrogen binding $\left(\Delta E_{H}=-146 \mathrm{meV}\right)$ at such induced carrier densities, which should further enhance the utility of this platform for understanding HER (and other reactions) at ultrathin electrodes.

\section{ASSOCIATED CONTENT}

\section{Supporting Information}

Materials; synthesis of monolayer $\mathrm{MoS}_{2}$; device fabrication; characterization: AFM image and Raman, photoluminescence, and XPS spectra of $\mathrm{MoS}_{2}$; electrical and electrochemical measurements: sheet conductance of $\mathrm{MoS}_{2}$ as a function of $V_{B G}$ (without electrolyte), sheet resistance of $\mathrm{MoS}_{2}$ measured in electrolyte simultaneously during electrochemical measurements, 
calculated in-plane polarization of a $\mathrm{MoS}_{2}$ electrode, polarization curves on a back-gated graphene electrode, determination of back-gate dielectric capacitance; computational methods.

The Supporting Information is available free of charge on the ACS Publications Website.

\section{AUTHOR INFORMATION}

\section{Corresponding Author}

*frisbie@umn.edu

\section{Notes}

The authors declare no competing financial interests.

\section{ACKNOWLEDGMENT}

The authors thank Dr. Chang-Hyun Kim for valuable discussions, Tony Whipple, Dr. Sushil Kumar Pandey and Dr. Youngdong Yoo for their help in the CVD synthesis, and Dr. Tao He, Dr. Zhuoran Zhang and Rui Ma for collecting AFM images. Y. W. acknowledges support through the Doctoral Dissertation Fellowship from the University of Minnesota. Parts of this work were carried out in the College of Science and Engineering Characterization Facility, University of Minnesota, which has received capital equipment funding from the NSF through the UMN MRSEC program under Award Number DMR-1420013, and in the Minnesota Nano Center which is supported by NSF through the National Nano Coordinated Infrastructure Network, Award Number NNCI -1542202. We would also like to thank the MSI Supercomputing Institute at the University of Minnesota for use of its computing resources.

\section{REFERENCES}

(1) Kim, C.-H.; Frisbie, C. D. Field Effect Modulation of Outer-Sphere Electrochemistry at Back-Gated, Ultrathin ZnO Electrodes. J. Am. Chem. Soc. 2016, 138 (23), 7220-7223. 
(2) Wang, Y.; Kim, C.-H.; Yoo, Y.; Johns, J. E.; Frisbie, C. D. Field Effect Modulation of Heterogeneous Charge Transfer Kinetics at Back-Gated Two-Dimensional MoS 2 Electrodes. Nano Lett. 2017, 17 (12), 7586-7592.

(3) Kim, C.-H.; Wang, Y.; Frisbie, C. D. Continuous and Reversible Tuning of Electrochemical Reaction Kinetics on Back-Gated 2D Semiconductor Electrodes: Steady-State Analysis Using a Hydrodynamic Method. Anal. Chem. 2019, 91 (2), 1627-1635.

(4) Sze, S. M.; Ng, K. K. Physics of Semiconductor Devices. In Physics of Semiconductor Devices; John Wiley \& Sons, Inc.: Hoboken, NJ, USA, 2006; pp 293-360.

(5) Jaramillo, T. F.; Jørgensen, K. P.; Bonde, J.; Nielsen, J. H.; Horch, S.; Chorkendorff, I. Identification of Active Edge Sites for Electrochemical $\mathrm{H}_{2}$ Evolution from $\mathrm{MoS}_{2}$ Nanocatalysts. Science 2007, 317 (5834), 100-102.

(6) Hinnemann, B.; Moses, P. G.; Bonde, J.; Jørgensen, K. P.; Nielsen, J. H.; Horch, S.; Chorkendorff, I.; Nørskov, J. K. Biomimetic Hydrogen Evolution: $\mathrm{MoS}_{2}$ Nanoparticles as Catalyst for Hydrogen Evolution. J. Am. Chem. Soc. 2005, 127 (15), 5308-5309.

(7) Li, H.; Tsai, C.; Koh, A. L.; Cai, L.; Contryman, A. W.; Fragapane, A. H.; Zhao, J.; Han, H. S.; Manoharan, H. C.; Abild-Pedersen, F.; Nørskov, J. K.; Zheng, X. Activating and Optimizing $\mathrm{MoS}_{2}$ Basal Planes for Hydrogen Evolution through the Formation of Strained Sulphur Vacancies. Nat. Mater. 2015, 15, 48.

(8) Li, G.; Zhang, D.; Qiao, Q.; Yu, Y.; Peterson, D.; Zafar, A.; Kumar, R.; Curtarolo, S.; Hunte, F.; Shannon, S.; Zhu, Y.; Yang, W.; Cao, L. All The Catalytic Active Sites of $\mathrm{MoS}_{2}$ for Hydrogen Evolution. J. Am. Chem. Soc. 2016, 138 (51), 16632-16638.

(9) Voiry, D.; Fullon, R.; Yang, J.; de Carvalho Castro e Silva, C.; Kappera, R.; Bozkurt, I.; Kaplan, D.; Lagos, M. J.; Batson, P. E.; Gupta, G.; Mohite, A. D.; Dong, L.; Er, D.; Shenoy, 
V. B.; Asefa, T.; Chhowalla, M. The Role of Electronic Coupling between Substrate and $2 \mathrm{DoS}_{2}$ Nanosheets in Electrocatalytic Production of Hydrogen. Nat. Mater. 2016, 15 (9), 1003-1009.

(10) Li, Y.; Wang, H.; Xie, L.; Liang, Y.; Hong, G.; Dai, H. MoS 2 Nanoparticles Grown on Graphene: An Advanced Catalyst for the Hydrogen Evolution Reaction. J. Am. Chem. Soc. 2011, 133, 7296.

(11) Wang, H.; Lu, Z.; Xu, S.; Kong, D.; Cha, J. J.; Zheng, G.; Hsu, P.-C.; Yan, K.; Bradshaw, D.; Prinz, F. B.; Cui, Y. Electrochemical Tuning of Vertically Aligned $\mathrm{MoS}_{2}$ Nanofilms and Its Application in Improving Hydrogen Evolution Reaction. Proc. Natl. Acad. Sci. 2013, $110(49), 19701-19706$.

(12) Voiry, D.; Salehi, M.; Silva, R.; Fujita, T.; Chen, M.; Asefa, T.; Shenoy, V. B.; Eda, G.; Chhowalla, M. Conducting $\mathrm{MoS}_{2}$ Nanosheets as Catalysts for Hydrogen Evolution Reaction. Nano Lett. 2013, 13 (12), 6222-6227.

(13) Lukowski, M. A.; Daniel, A. S.; Meng, F.; Forticaux, A.; Li, L.; Jin, S. Enhanced Hydrogen Evolution Catalysis from Chemically Exfoliated Metallic $\mathrm{MoS}_{2}$ Nanosheets. J. Am. Chem. Soc. 2013, 135 (28), 10274-10277.

(14) Wang, H.; Lu, Z.; Kong, D.; Sun, J.; Hymel, T. M.; Cui, Y. Electrochemical Tuning of $\mathrm{MoS}_{2}$ Nanoparticles on Three-Dimensional Substrate for Efficient Hydrogen Evolution. ACS Nano 2014, 8 (5), 4940-4947.

(15) Tsai, C.; Li, H.; Park, S.; Park, J.; Han, H. S.; Nørskov, J. K.; Zheng, X.; Abild-Pedersen, F. Electrochemical Generation of Sulfur Vacancies in the Basal Plane of $\mathrm{MoS}_{2}$ for Hydrogen Evolution. Nat. Commun. 2017, 8, 15113.

(16) Deng, J.; Li, H.; Xiao, J.; Tu, Y.; Deng, D.; Yang, H.; Tian, H.; Li, J.; Ren, P.; Bao, X. 
Triggering the Electrocatalytic Hydrogen Evolution Activity of the Inert Two-Dimensional $\mathrm{MoS}_{2}$ Surface via Single-Atom Metal Doping. Energy Environ. Sci. 2015, 8(5), 1594-1601.

(17) Shi, Y.; Zhou, Y.; Yang, D.-R.; Xu, W.-X.; Wang, C.; Wang, F.-B.; Xu, J.-J.; Xia, X.-H.; Chen, H.-Y. Energy Level Engineering of $\mathrm{MoS}_{2}$ by Transition-Metal Doping for Accelerating Hydrogen Evolution Reaction. J. Am. Chem. Soc. 2017, 139 (43), 1547915485.

(18) Benson, E. E.; Zhang, H.; Schuman, S. A.; Nanayakkara, S. U.; Bronstein, N. D.; Ferrere, S.; Blackburn, J. L.; Miller, E. M. Balancing the Hydrogen Evolution Reaction, Surface Energetics, and Stability of Metallic $\mathrm{MoS}_{2}$ Nanosheets via Covalent Functionalization. $J$. Am. Chem. Soc. 2018, 140 (1), 441-450.

(19) Wang, J.; Yan, M.; Zhao, K.; Liao, X.; Wang, P.; Pan, X.; Yang, W.; Mai, L. Field Effect Enhanced Hydrogen Evolution Reaction of $\mathrm{MoS}_{2}$ Nanosheets. Adv. Mater. 2017, 29 (7), 1604464-n/a.

(20) Yan, M.; Pan, X.; Wang, P.; Chen, F.; He, L.; Jiang, G.; Wang, J.; Liu, J. Z.; Xu, X.; Liao, X.; Yang, J.; Mai, L. Field-Effect Tuned Adsorption Dynamics of $\mathrm{VSe}_{2}$ Nanosheets for Enhanced Hydrogen Evolution Reaction. Nano Lett. 2017, 17 (7), 4109-4115.

(21) Yoo, Y.; Degregorio, Z. P.; Johns, J. E. Seed Crystal Homogeneity Controls Lateral and Vertical Heteroepitaxy of Monolayer $\mathrm{MoS}_{2}$ and $\mathrm{WS}_{2}$. J. Am. Chem. Soc. 2015, 137 (45), $14281-14287$.

(22) Yu, Y.; Li, C.; Liu, Y.; Su, L.; Zhang, Y.; Cao, L. Controlled Scalable Synthesis of Uniform, High-Quality Monolayer and Few-Layer $\mathrm{MoS}_{2}$ Films. Sci. Rep. 2013, 3, 1866.

(23) Yu, Y.; Huang, S.-Y.; Li, Y.; Steinmann, S. N.; Yang, W.; Cao, L. Layer-Dependent Electrocatalysis of $\mathrm{MoS}_{2}$ for Hydrogen Evolution. Nano Lett. 2014, 14 (2), 553-558. 
(24) Li, G.; Zhang, D.; Yu, Y.; Huang, S.; Yang, W.; Cao, L. Activating MoS 2 for PH-Universal Hydrogen Evolution Catalysis. J. Am. Chem. Soc. 2017, 139 (45), 16194-16200.

(25) Seh, Z. W.; Kibsgaard, J.; Dickens, C. F.; Chorkendorff, I.; Nørskov, J. K.; Jaramillo, T. F. Combining Theory and Experiment in Electrocatalysis: Insights into Materials Design. Science (80-. ). 2017, 355 (6321), eaad4998.

(26) Shinagawa, T.; Garcia-Esparza, A. T.; Takanabe, K. Insight on Tafel Slopes from a Microkinetic Analysis of Aqueous Electrocatalysis for Energy Conversion. Sci. Rep. 2015, 5 (September), 1-21.

(27) Parsons, R. The Rate of Electrolytic Hydrogen Evolution and the Heat of Adsorption of Hydrogen. Trans. Faraday Soc. 1958, 54, 1053.

(28) Nørskov, J. K.; Bligaard, T.; Logadottir, A.; Kitchin, J. R.; Chen, J. G.; Pandelov, S.; Stimming, U. Trends in the Exchange Current for Hydrogen Evolution. J. Electrochem. Soc. 2005, $152(3)$, J23.

(29) Trasatti, S. Work Function, Electronegativity, and Electrochemical Behaviour of Metals: III. Electrolytic Hydrogen Evolution in Acid Solutions. J. Electroanal. Chem. Interfacial Electrochem. 1972, 39 (1), 163-184.

(30) Sheng, W.; Myint, M.; Chen, J. G.; Yan, Y. Correlating the Hydrogen Evolution Reaction Activity in Alkaline Electrolytes with the Hydrogen Binding Energy on Monometallic Surfaces. Energy Environ. Sci. 2013, 6 (5), 1509-1512.

(31) Zhang, Y.; Kolmakov, A.; Chretien, S.; Metiu, H.; Moskovits, M. Control of Catalytic Reactions at the Surface of a Metal Oxide Nanowire by Manipulating Electron Density Inside It. Nano Lett. 2004, 4 (3), 403-407.

(32) Baker, L. R.; Hervier, A.; Kennedy, G.; Somorjai, G. A. Solid-State Charge-Based Device 
for Control of Catalytic Carbon Monoxide Oxidation on Platinum Nanofilms Using External Bias and Light. Nano Lett. 2012, 12 (5), 2554-2558. 

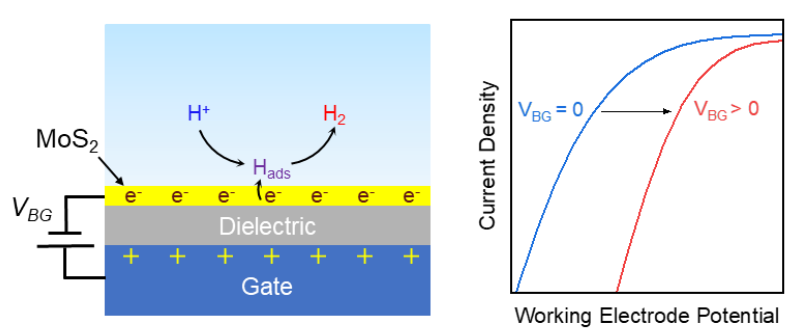


\section{Supporting Information}

\section{Field Effect Modulation of Electrocatalytic Hydrogen Evolution at Back-Gated Two-Dimensional $\mathrm{MoS}_{2}$ Electrodes}

Yan Wang ${ }^{\dagger}$, Sagar Udyavara ${ }^{*}$ Matthew Neurock ${ }^{\star}$ and, C. Daniel Frisbie ${ }^{* *}$

\$Department of Chemical Engineering and Materials Science, University of Minnesota, 421

Washington Avenue SE, Minneapolis, Minnesota 55455, United States

$\dagger$ Department of Chemistry, University of Minnesota, 207 Pleasant Street SE, Minneapolis, Minnesota 55455, United States

*Corresponding Author. Phone: (+1) 612-625-0779; Email: frisbie@umn.edu 


\section{S1. Materials}

Sulfuric acid $\left(\mathrm{H}_{2} \mathrm{SO}_{4}, 95-98 \%\right.$, ACS grade), was purchased form BDH ARISTAR. Water used to prepare aqueous solutions was purchased from Fisher Chemical. P-type highly boron doped silicon wafers with thermally grown 300 -nm-thick $\mathrm{SiO}_{2}\left(\mathrm{SiO}_{2} / p-\mathrm{Si}\right)$, used in both chemical vapor deposition (CVD) and device fabrication, were purchased from Silicon Valley Microelectronics. Molybdenum oxide $\left(\mathrm{MoO}_{3}, 99.97 \%\right)$, sulfur powder (99.98\%), molybdenum sulfide powder (particle size $<2 \mu \mathrm{m}, 99 \%$ ), ammonium persulfate $\left(\left(\mathrm{NH}_{4}\right)_{2} \mathrm{~S}_{2} \mathrm{O}_{8}, 98 \%\right.$, reagent grade), and poly(methyl methacrylate) (PMMA, molecular weight: $350 \mathrm{kDa}$ ) were purchased from Sigma-Aldrich. Polydimethylsiloxane (PDMS) precursor and its curing agent (Sylgard 184 silicone elastomer kit) were purchased from Dow Corning. $\mathrm{Ag} / \mathrm{AgCl}$ reference electrodes (CHI111P) were purchased from Fisher Scientific. Graphite rods (99.95\%) with a diameter of 1.0 $\mathrm{mm}$ used as counter electrodes were purchased from Sigma-Aldrich. CVD monolayer graphene grown on $\mathrm{Cu}$ foil was purchased from Graphene Square Inc. (Seoul, South Korea).

\section{S2. Synthesis of Monolayer $\mathrm{MoS}_{2}$}

Monolayer $\mathrm{MoS}_{2}$ was synthesized by $\mathrm{CVD}$ using $\mathrm{MoO}_{3}$ and sulfur as precursors in a horizontal hot-wall reactor system (PlanarTECH, Planar GROW-5S). In a typical CVD growth, an alumina boat $(50 \mathrm{~mm} \times 12 \mathrm{~mm} \times 10 \mathrm{~mm})$ containing $0.5 \mathrm{mg} \mathrm{MoO}_{3}$ powder was placed at the center of the hot-wall furnace. Another alumina boat of same size, holding $200 \mathrm{mg}$ sulfur powder, was placed upstream of the furnace, $25 \mathrm{~cm}$ away from the furnace center. A receiving substrate $\left(\mathrm{SiO}_{2} / p\right.$ - $\left.\mathrm{Si}, 30 \times 30 \mathrm{~mm}^{2}\right)$ was mounted face-down on the $\mathrm{MoO}_{3}$-containing boat. At room temperature, the quartz tube was first pumped down to vacuum and then purged by flowing Ar gas at a flow rate of $500 \mathrm{sccm}$ for about $10 \mathrm{~min}$ until the pressure reached 760 torr again. 
After purging, the furnace temperature was ramped to $850{ }^{\circ} \mathrm{C}$ in 30 min and was kept at $850{ }^{\circ} \mathrm{C}$ for $15 \mathrm{~min}$, while the flow rate of Ar gas was held at $67.5 \mathrm{sccm}$. At $850{ }^{\circ} \mathrm{C}$ furnace temperature, the temperature of sulfur precursor was measured to be approximately $300{ }^{\circ} \mathrm{C}$. After growth, the furnace was first naturally cooled to $650{ }^{\circ} \mathrm{C}$ and then was moved to the downstream side to cool the receiving substrate rapidly to room temperature.

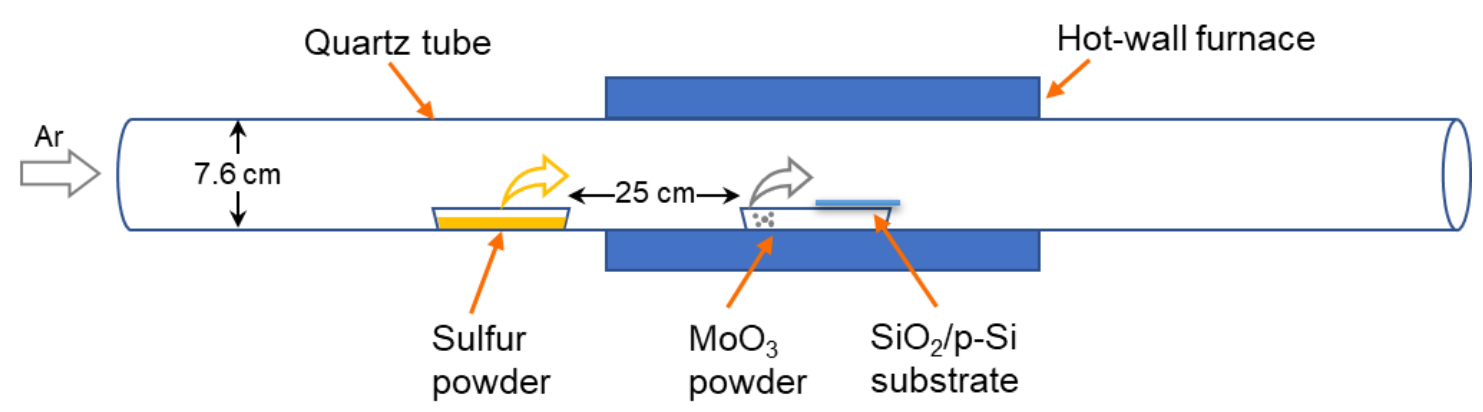

Figure S1. Schematic illustration of the CVD setup used in this study

\section{S3. Device Fabrication}

The fabrication process was described in detail in our prior work. ${ }^{1,2}$ Briefly, the as-grown $\mathrm{MoS}_{2}$ was spin-coated with PMMA and the resulting PMMA film holding $\mathrm{MoS}_{2}$ flakes was separated from the growth substrate by etching the interfacial $\mathrm{SiO}_{2}$ layer with $\mathrm{KOH}$ solution (aqueous, $30 \mathrm{wt} \%$ ) and then transferred onto a $\mathrm{SiO}_{2} / p-\mathrm{Si}$ substrate that is used as the dielectric/back gate stack. After removing PMMA with acetone, the $\mathrm{MoS}_{2}$ flake with a desirable size and minimal cracks was patterned into a rectangular stripe by selective $\mathrm{O}_{2}$ plasma etching following photolithography. Another step of photolithography was carried out before e-beam evaporation of $\mathrm{Ti} / \mathrm{Au}(3 \mathrm{~nm} / 30 \mathrm{~nm})$ metal contacts on $\mathrm{MoS}_{2}$. To prevent electrochemical reactions from occurring on the surface of $\mathrm{Au}$, a layer of $30 \mathrm{~nm}$ thick $\mathrm{SiO}_{2}$ was e-beam evaporated on top of the peripheral metal area of $\mathrm{MoS}_{2}$, with the help of a shadow mask. After 
lift-off, the fabricated $\mathrm{MoS}_{2}$ working electrode was ready to assemble with a molded PDMS microfluidic flow cell. Similarly, graphene electrodes were prepared through these steps described above, except the etchant solution used to separate the PMMA film holding graphene from $\mathrm{Cu}$ foil in the transfer step was $100 \mathrm{~g} / \mathrm{L}$ ammonium persulfate aqueous solution. $\mathrm{Pt}$ electrodes were prepared via photolithography and e-beam evaporation of Ti/Pt $(3 \mathrm{~nm} / 50 \mathrm{~nm})$ on $\mathrm{SiO}_{2} / p$-Si wafers.

The master mold for PDMS microfluidic channels $(200-\mu \mathrm{m}$ wide, $50-\mu \mathrm{m}$ tall $)$ was fabricated on a 4-inch Si wafer with a negative photoresist, SU8 2050 (MicroChem), via photolithography. ${ }^{2}$ PDMS precursor mixed with its curing agent (10:1 by weight) was poured onto the master mold and cured at $70{ }^{\circ} \mathrm{C}$ in an oven for 3 to $4 \mathrm{~h}$. The cured PDMS was cut into separate blocks where the inlet and outlet holes were made with 1- and 3-mm diameter punch tools, respectively. A molded PDMS flow cell was bonded onto a back-gated $\mathrm{MoS}_{2}$ electrode substrate through a condensation reaction with $\mathrm{SiO}_{2}$ at $120{ }^{\circ} \mathrm{C}$, following $\mathrm{O}_{2}$ plasma treatment of the bonding surfaces ${ }^{2}$ (note that $\mathrm{MoS}_{2}$ was covered by a shadow mask in this $\mathrm{O}_{2}$ plasma treatment).

\section{S4. Characterization}

Raman and photoluminescence spectra were obtained with a $532 \mathrm{~nm}$ laser using a confocal Raman spectrometer (Witec Aphas300R). Atomic force microscopy (AFM) images were collected in the contact mode on a scanning probe microscope (Bruker Nanoscope $\mathrm{V}$ Multimode 8). X-ray photoelectron spectroscopy (XPS) measurements were carried out in ultrahigh vacuum $\left(10^{-7}\right.$ pascal) with an $\mathrm{Al} \mathrm{K \alpha} \mathrm{X}$-ray monochromatic source $(1486.6 \mathrm{eV})$ on a PHI VersaProbe III scanning XPS Microprobe. 

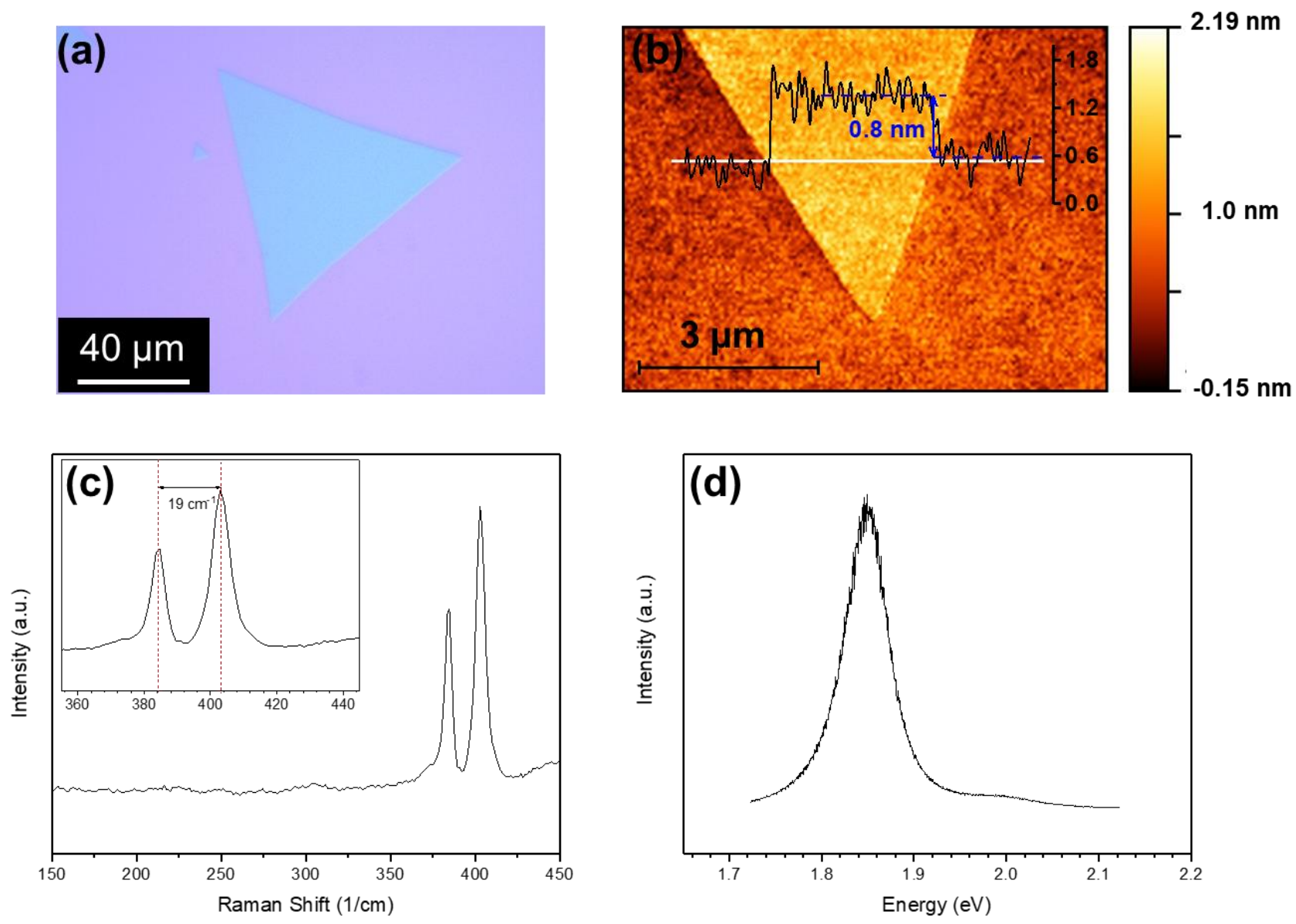

Figure S2. (a) Optical image of CVD grown $\mathrm{MoS}_{2}$ supported on $\mathrm{SiO}_{2} / p$-Si substrate; (b) AFM image obtained at the edge of a $\mathrm{MoS}_{2}$ flake; The inset of (b) shows the height profile measured along the white line; (c) Raman and (d) photoluminescence spectra collected on the large $\mathrm{MoS}_{2}$ flake in (a). 

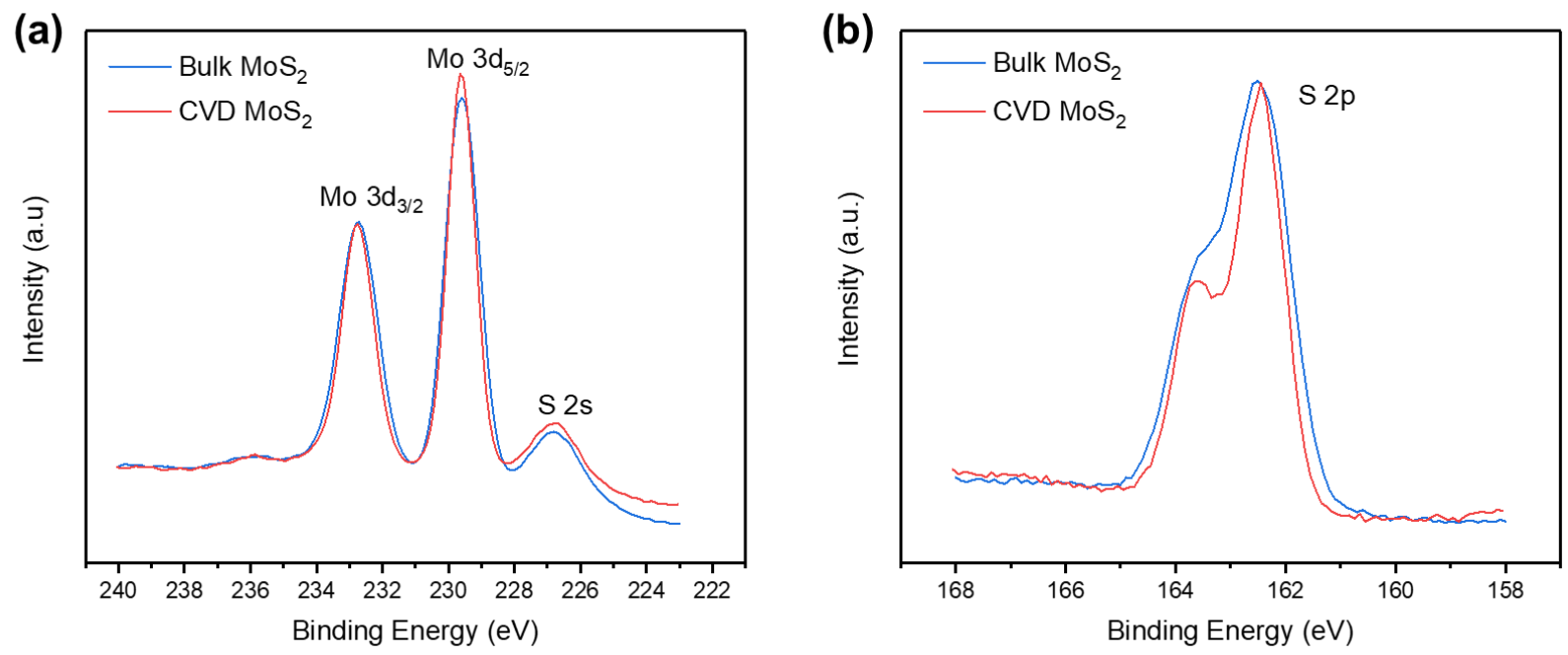

Figure S3. High-resolution X-ray photoelectron spectra of CVD monolayer $\mathrm{MoS}_{2}$ flakes and bulk MoS 2 powder: (a) Mo 3d states and (b) S 2p states. The high-resolution Mo 3d and S 2p peaks shown in (a) and (b) were used to calculate the $\mathrm{S} / \mathrm{Mo}$ ratios. The $\mathrm{S}$ vacancy concentration of CVD monolayer $\mathrm{MoS}_{2}$ samples was estimated to be $\sim 6 \%$ by comparing the $\mathrm{S} / \mathrm{Mo}$ atomic ratio of CVD monolayer $\mathrm{MoS}_{2}$ to that of bulk $\mathrm{MoS}_{2}$ powder. 

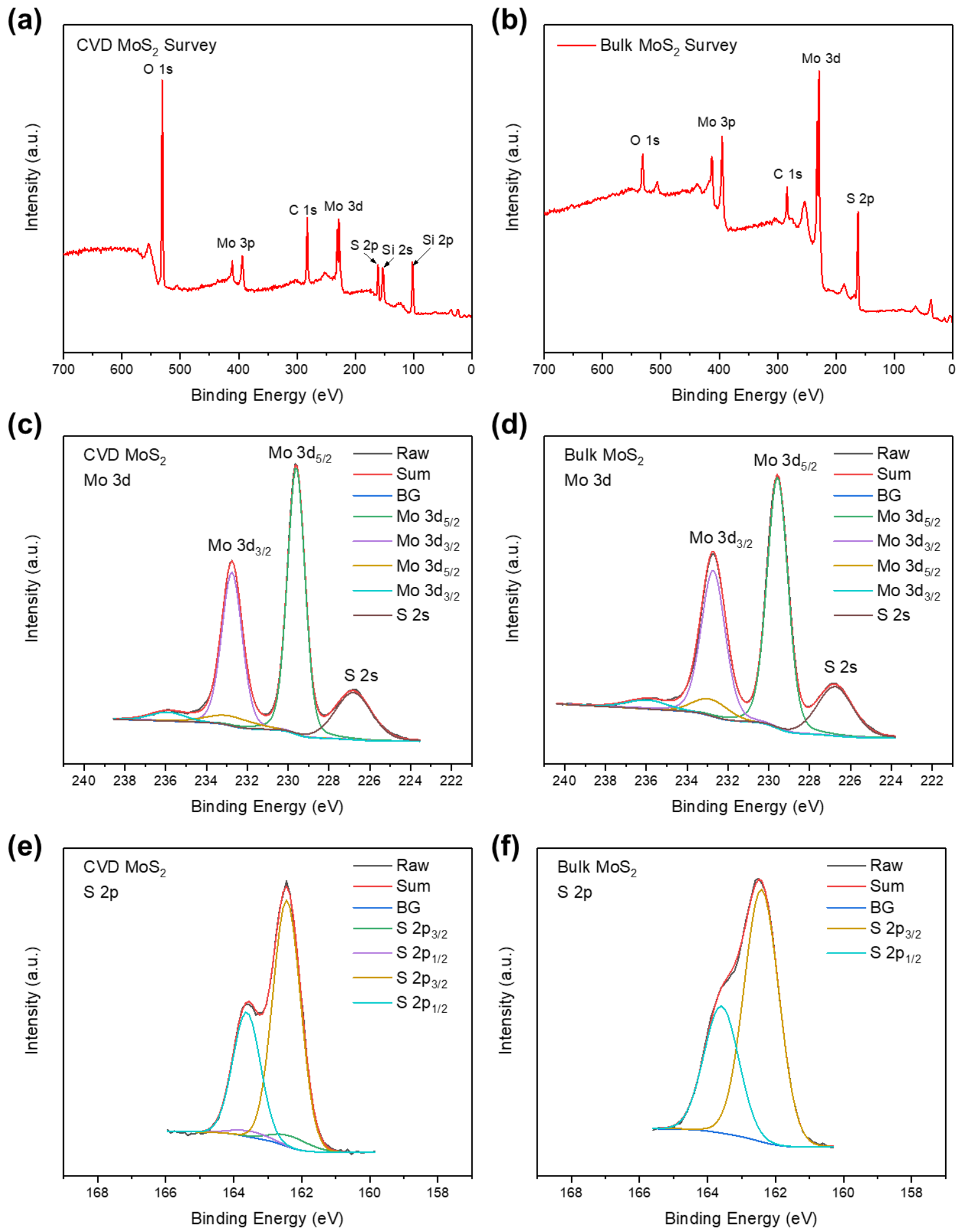

Figure S4. (a) and (b) X-ray photoelectron spectroscopy (XPS) survey spectra taken from 0 to $700 \mathrm{eV}$; (c) and (d) Mo 3d raw XPS spectra and fitted curves; (e) and (f) S 2p raw XPS spectra and fitted curves. (a), (c) and (e) are for CVD monolayer $\mathrm{MoS}_{2}$ samples on $\mathrm{SiO}_{2} / \mathrm{Si}$. (b), (d) and (f) are for bulk $\mathrm{MoS}_{2}$ powder on conductive aluminum foil tape. 


\section{S5. Electrical and Electrochemical Measurements}

The electrical and electrochemical configuration used in experiments is shown in Figure 1b. To obtain $\mathrm{MoS}_{2}$ sheet conductance as a function of back-gate bias $V_{B G}$ without electrolyte, Keithley 2400 and 2611 source meters were used to apply $V_{B G}$ and source-to-drain bias $V_{S D}$, respectively, while measuring currents. To perform electrochemical experiments while testing sheet conductance of $\mathrm{MoS}_{2}$, an $\mathrm{Ag} / \mathrm{AgCl}$ reference electrode and a graphite rod counter electrode were introduced to the setup. The $\mathrm{Ag} / \mathrm{AgCl}$ (filled with $1 \mathrm{M} \mathrm{KCl}$ ) reference electrode was plugged into a Luggin capillary (filled with $0.5 \mathrm{M} \mathrm{H}_{2} \mathrm{SO}_{4}$ ) whose tip was placed at the outlet of the flow cell. The graphite counter electrode was inserted 3 to $5 \mathrm{~mm}$ away from the outlet. The $\mathrm{MoS}_{2}$ working electrode was aligned in the microfluidic channel and placed $\sim 100 \mu \mathrm{m}$ away from the outlet, as shown in Figure 1b. During the sweep of working electrode potential, the source metal contact on the $\mathrm{MoS}_{2}$ working electrode was grounded while a $5 \mathrm{mV}$ bias was applied at the drain metal contact. The reaction current was obtained by adding up the currents measured at both source and drain contacts (detailed explanation can be found in our prior reports ${ }^{2,3}$ ). We used four source meters (Keithley 2400, 2611, 2612 and 236) to apply potentials and measure currents and data were collected with an in-house LabView program. The working electrode potential $V_{W}$ was iR corrected to account for ohmic losses in the electrolyte using the same method as reported before, ${ }^{2}$ where a value of $211 \mathrm{mS} / \mathrm{cm}$ found in the literature ${ }^{4}$ was used as the bulk conductivity of $0.5 \mathrm{M} \mathrm{H}_{2} \mathrm{SO}_{4}$. All the electrical and electrochemical measurements were conducted in ambient environment at room temperature. 


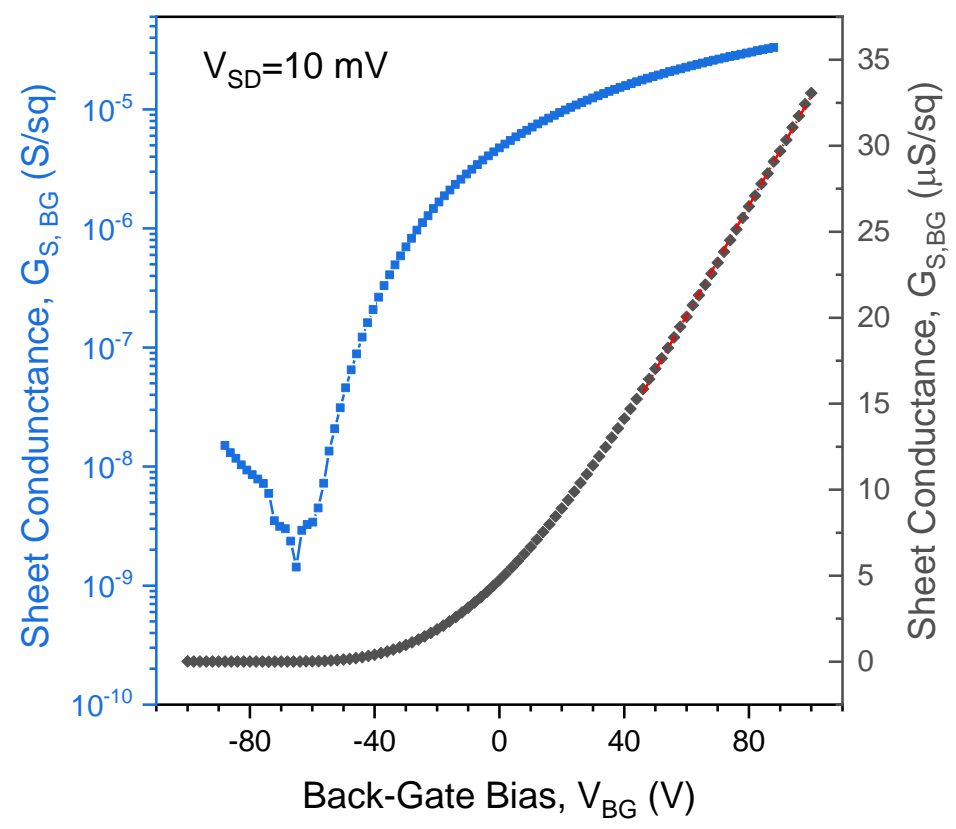

Figure S5. Sheet conductance, $G_{S}$, of a representative $\mathrm{MoS}_{2}$ electrode $\left(50 \times 50 \mathrm{um}^{2}\right)$ measured by applying a constant source-to-drain bias, $V_{S D}=10 \mathrm{mV}$, while sweeping back-gate bias $V_{B G}$ from -100 to $+100 \mathrm{~V}$ at a sweep rate of $2 \mathrm{~V} / \mathrm{s}$. The blue and gray plots are shown in semilog and linear scale, respectively. The sheet conductance was obtained without any electrolyte or encapsulation layer on top of $\mathrm{MoS}_{2}$. The field effect electron mobility $\mu$ was calculated using the following equation: $\mu=\frac{d G_{S}}{d V_{B G}} \cdot \frac{1}{C_{B G}}$, where $C_{B G}$ is the specific capacitance (capacitance per unit area) of 300nm-thick $\mathrm{SiO}_{2}$ dielectric layer, which was estimated to be $15 \mathrm{nF} / \mathrm{cm}^{2}$ via displacement measurements (see Figure S8). The slope of the red dashed line shown here was used as $\frac{d G_{S}}{d V_{B G}}$ to calculate $\mu$. 
(a)

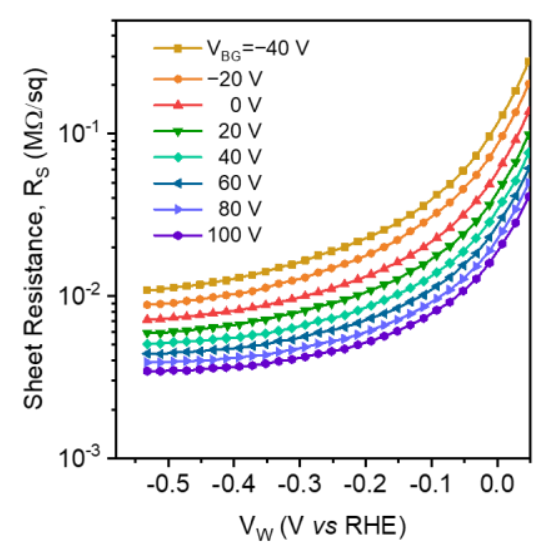

(b)

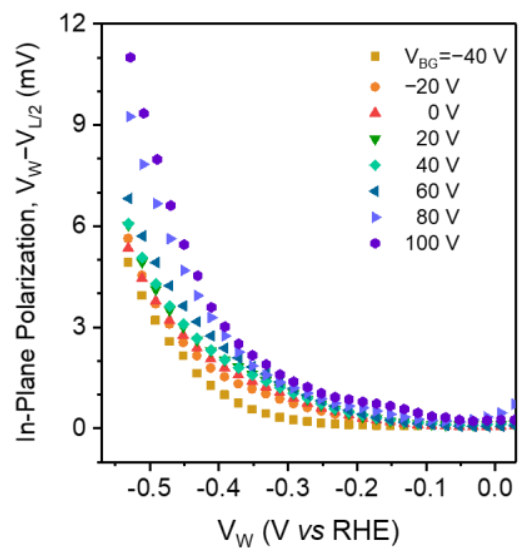

(c)

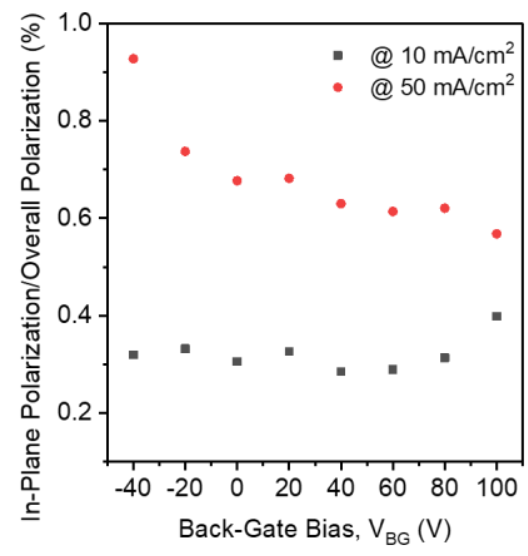

Figure S6. (a) Sheet resistance, $R_{S}$, of a back-gated $\mathrm{MoS}_{2}$ electrode $\left(50 \times 50 \mu \mathrm{m}^{2}\right)$ simultaneously obtained during sweep of working electrode potential $V_{W}$ in $0.5 \mathrm{M} \mathrm{H}_{2} \mathrm{SO}_{4}$ at different back gate biases $V_{B G}$ (Note that a source-to-drain bias $V_{S D}=5 \mathrm{mV}$ was applied to measure $R_{S}$ ); (b) corresponding in-plane polarization calculated (The method used to calculate in-plane polarization was described in detail in our previous report. ${ }^{3}$ ); (c) percentage of in-plane polarization to overall polarization at current density $i_{w}=10$ and $50 \mathrm{~mA} / \mathrm{cm}^{2}$ as a function of applied back-gate bias $V_{B G}$. 


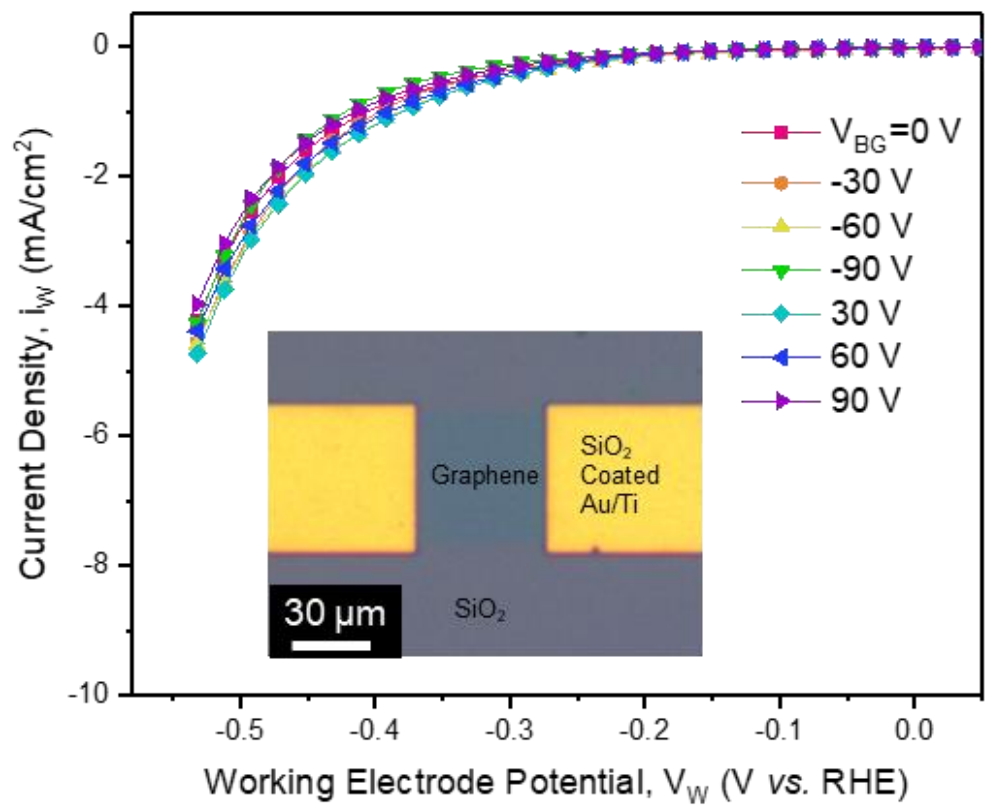

Figure S7. Polarization curves obtained in flowing $0.5 \mathrm{M} \mathrm{H}_{2} \mathrm{SO}_{4}$ on a back-gated graphene electrode $\left(50 \times 50 \mu \mathrm{m}^{2}\right)$ at varying back-gate biases $V_{B G}$. The inset optical microscopy image shows a plan view of a back-gated graphene electrode. The scan rate of the working electrode potential is $50 \mathrm{mV} / \mathrm{s}$ and the volumetric flow of the electrolyte is $10 \mu \mathrm{L} / \mathrm{min}$. 

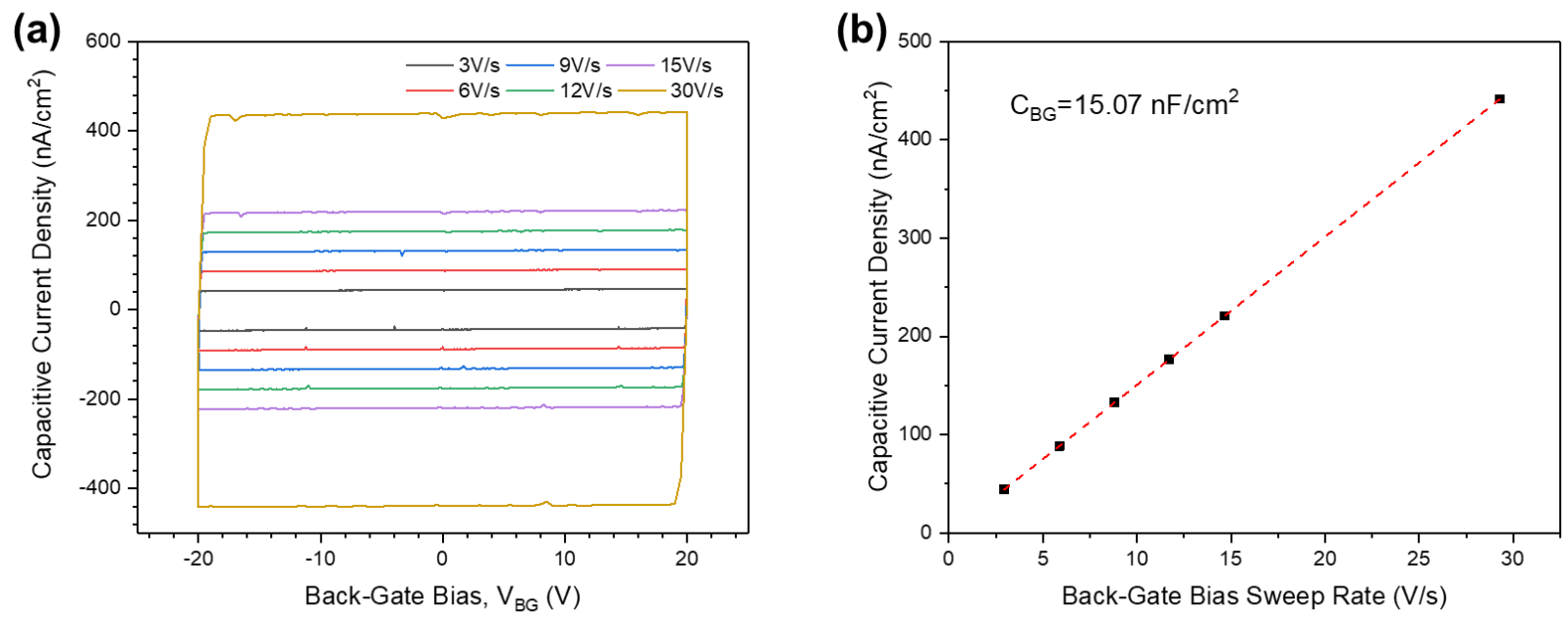

Figure S8. (a) Capacitive current density measured by sweeping back-gate bias $V_{B G}$ applied on an $\mathrm{Au} / \mathrm{SiO}_{2} / p$-Si structure at different $V_{B G}$ sweep rates; (b) Averaged capacitive current density as a function of $V_{B G}$ scan rates in (a). A $9 \mathrm{~mm}^{2}$ gold electrode was used in this measurement and it was prepared in a similar procedure as described in S3 via photolithography and e-beam evaporation of $\mathrm{Ti} / \mathrm{Au}(3 \mathrm{~nm} / 30 \mathrm{~nm}$ thick). 


\section{S6. Computational Methods}

Spin polarized density functional theory (DFT) calculations reported herein were carried out using plane wave DFT code implemented in Vienna Ab-initio Simulation Package (VASP). ${ }^{5-}$ ${ }^{8}$ All of the calculations presented herein were carried using nonlocal gradient calculations using the RPBE functional within the Generalized Gradient Approximation (GGA) to determine the exchange and correlation gradient corrections. ${ }^{9}$ PAW pseudopotentials were used to describe the core electrons and their interaction with the valence electrons. ${ }^{10,11}$ An optimized lattice constant of a $=3.19 \AA$ was computed for the $2 \mathrm{H}$ phase of bulk $\mathrm{MoS}_{2}$ which is in agreement with the experimentally determined value of $\mathrm{a}=3.16 \AA \mathrm{A}^{12} \mathrm{~A} 3 \times 3$ supercell of monolayer $\mathrm{MoS}_{2}$ was then constructed by cleaving along the (001) plane of the $2 \mathrm{H}-\mathrm{MoS}_{2}$ crystal. A vacuum space of $20 \AA$ was employed for the calculations to minimize interaction between periodic layers. This monolayer $\mathrm{MoS}_{2}$ surface with a surface area of $79.31 \AA^{2}$ was then used as the model for our calculations. An energy cutoff of $1000 \mathrm{eV}$ was used to construct the plane waves for the DFT calculations. The structural optimizations were carried out until the maximum force on each of the ions was less than $0.05 \mathrm{eV} / \mathrm{A}$. A tolerance of $10^{-6} \mathrm{eV}$ was specified for the convergence of the SCF cycle. A 3x3x1 gamma-centered k-point grid was used for structural optimizations, while a 6x6x1 k-point grid was used for energy calculations. The band structure was calculated along the high symmetry k-point path of $\Gamma-\mathrm{M}-\mathrm{K}-\Gamma$ for $\mathrm{MoS}_{2}$ with 25 intersections between each of the kpoints. The $\mathrm{S}$ vacancy was created by removing a $\mathrm{S}$ atom from the pristine monolayer $\mathrm{MoS}_{2}$ surface, thus generating a $5.55 \% \mathrm{~S}$ vacancy concentration which results in the formation of the mid-gap states shown in Figure S9 which is consistent with the states previously reported for vacancies in the monolayer surfaces of $\mathrm{MoS}_{2} \cdot{ }^{13}$ These mid gap states consist mainly of Mo dstates (Figure S10). 
Hydrogen adsorption studies examined the binding of atomic $\mathrm{H}$ to the $\mathrm{S}$ vacancy on the $\mathrm{MoS}_{2}$ surface as shown in Figure S11. Previous reports suggest that these S vacancies serve as the active sites for HER on $\mathrm{MoS}_{2 .}{ }^{13}$ To examine the effect of gate bias, the system was charged with excess electrons depending on the excess charge carrier density determined experimentally corresponding to the applied bias, summarized in Table S1. The hydrogen binding energy was calculated as a function charge using the following equation:

$$
\Delta \mathrm{E}_{\mathrm{H}}=\mathrm{E}\left(\mathrm{MoS}_{2}-\mathrm{H}\right)(\mathrm{q})-\mathrm{E}\left(\mathrm{MoS}_{2}\right)(\mathrm{q})-0.5 \mathrm{E}\left(\mathrm{H}_{2}\right)(\mathrm{q}=0)
$$

where $\mathrm{E}\left(\mathrm{MoS}_{2}-\mathrm{H}\right)(\mathrm{q})$ is the energy of the model $\mathrm{MoS}_{2}$ surface used with the $\mathrm{H}$ adsorbed on the $\mathrm{S}$ vacancy site at charge $\mathrm{q}, \mathrm{E}\left(\mathrm{MoS}_{2}\right)$ (q) is the energy of the model $\mathrm{MoS}_{2}$ used without adsorbed $\mathrm{H}$ at charge $\mathrm{q}$ and $\mathrm{E}\left(\mathrm{H}_{2}\right)$ is the energy of the hydrogen molecule.

The calculated free energies $\left(\Delta \mathrm{G}_{\mathrm{H}}=\Delta \mathrm{E}_{\mathrm{H}}+\Delta \mathrm{ZPE}-\mathrm{T} \Delta \mathrm{S}\right)$ are known to be good descriptors for the HER activity. ${ }^{15}$ In the present study, the changes in ZPE $(\triangle \mathrm{ZPE})$ and $\mathrm{S}(\Delta \mathrm{S})$ aren't expected to change at different charged conditions. As such, we use $\Delta \mathrm{E}_{\mathrm{H}}$ to provide the important qualitative trends as was described above.

Table S1. Number of excess electrons used to charge the system corresponding to the different voltage bias and excess charge carrier density

\begin{tabular}{|c|c|c|}
\hline $\begin{array}{c}\text { Back-gate } \\
\text { voltage }\left(\mathrm{V}_{\mathrm{BG}}\right)\end{array}$ & $\begin{array}{c}\text { Excess charge carrier } \\
\text { density }\left(\mathrm{e}^{-} / \mathrm{cm}^{2}\right)\end{array}$ & $\begin{array}{c}\text { Excess electrons added } \\
\text { to the model system }\left(\mathrm{e}^{-}\right)\end{array}$ \\
\hline 0 & 0 & 0 \\
\hline 20 & $2 \times 10^{12}$ & 0.016 \\
\hline 40 & $4 \times 10^{12}$ & 0.032 \\
\hline 60 & $6 \times 10^{12}$ & 0.048 \\
\hline 80 & $8 \times 10^{12}$ & 0.064 \\
\hline 100 & $10^{13}$ & 0.08 \\
\hline
\end{tabular}



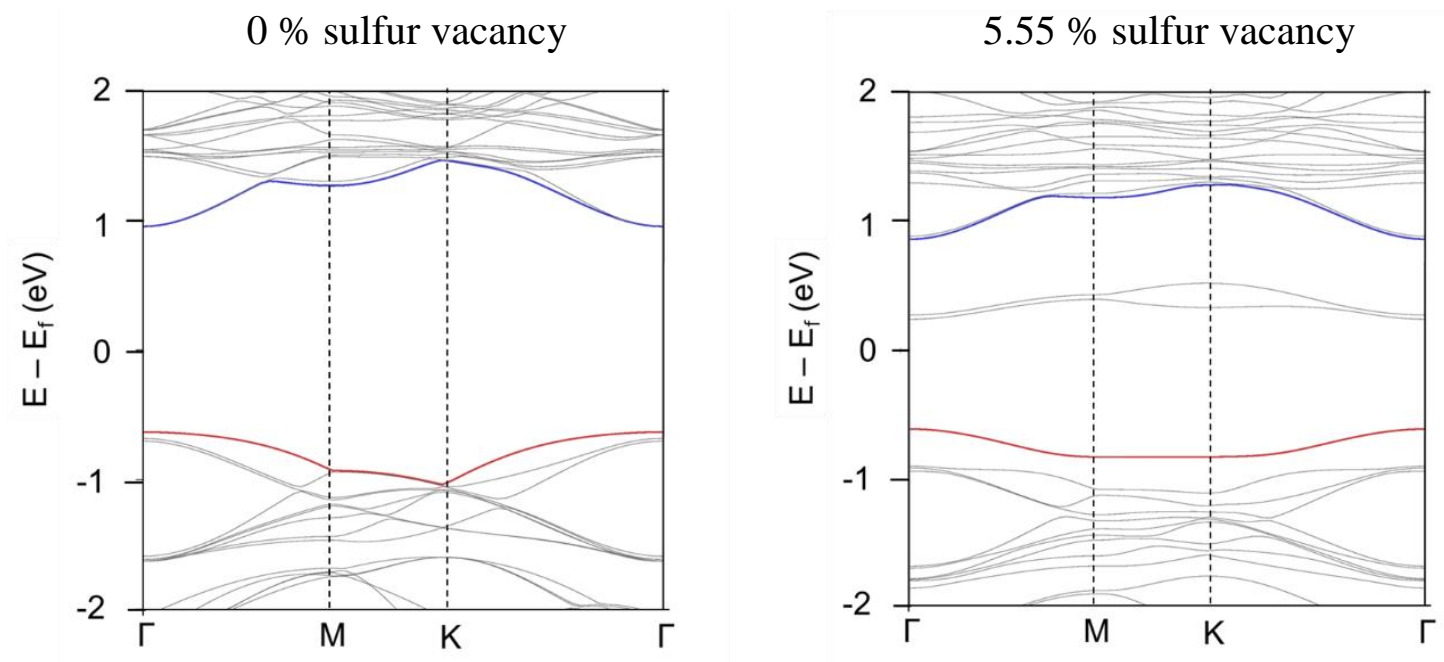

Figure S9. Band structures of pristine $\mathrm{MoS}_{2}$ with $0 \% \mathrm{~S}$ vacancy and $\mathrm{MoS}_{2}$ with $5.55 \% \mathrm{~S}$ vacancy showing introduction of mid-gap states at zero system charge.

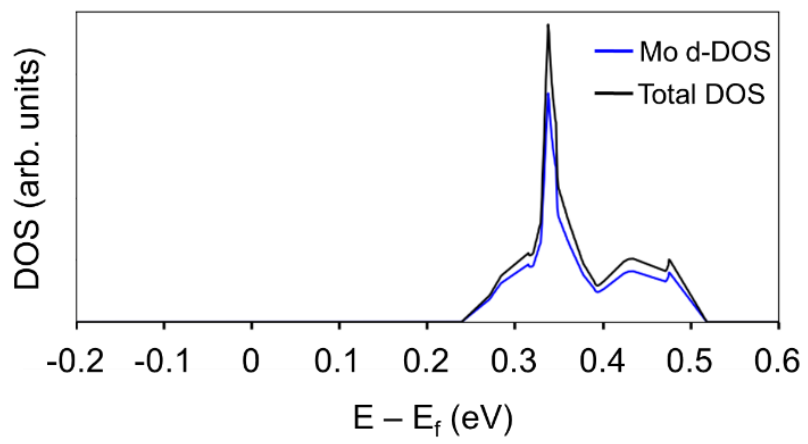

Figure S10. Projected Mo d-density of states and total density of states of $\mathrm{MoS}_{2}$ surface with $5.55 \% \mathrm{~S}$ vacancy at zero charge. 


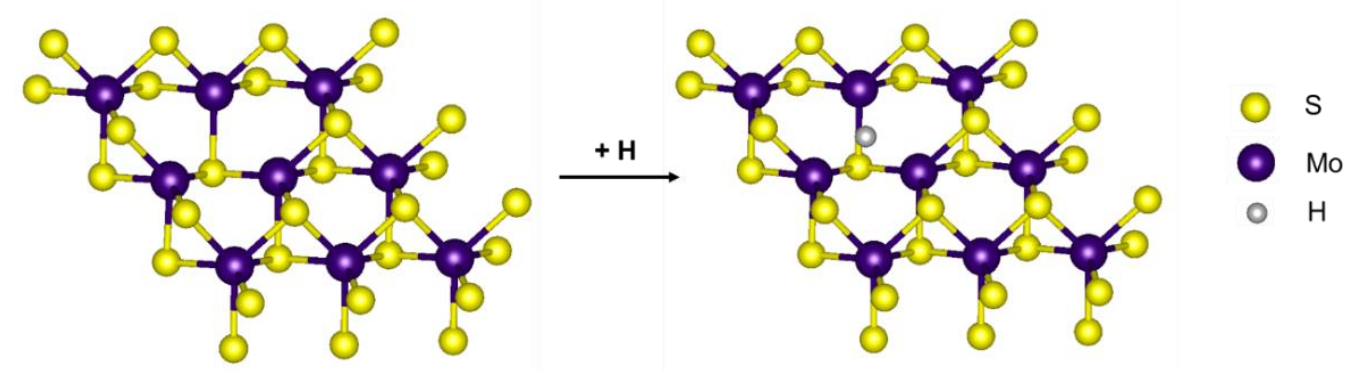

Figure S11. Hydrogen adsorption on S vacancy site.

\section{References}

(1) Wang, Y.; Kim, C.-H.; Yoo, Y.; Johns, J. E.; Frisbie, C. D. Field Effect Modulation of Heterogeneous Charge Transfer Kinetics at Back-Gated Two-Dimensional MoS Electrodes. Nano Lett. 2017, 17 (12), 7586-7592.

(2) Kim, C.-H.; Wang, Y.; Frisbie, C. D. Continuous and Reversible Tuning of Electrochemical Reaction Kinetics on Back-Gated 2D Semiconductor Electrodes: SteadyState Analysis Using a Hydrodynamic Method. Anal. Chem. 2019, 91 (2), 1627-1635.

(3) Kim, C.-H.; Frisbie, C. D. Field Effect Modulation of Outer-Sphere Electrochemistry at Back-Gated, Ultrathin ZnO Electrodes. J. Am. Chem. Soc. 2016, 138 (23), 7220-7223.

(4) CRC Handbook of Chemistry and Physics, 70th edition; Weast, R. C., Ed.; CRC Press: Boca Raton, FL, 1989.

(5) Kresse, G. \& Hafner, J. Ab initio molecular dynamics for liquid metals. Phys. Rev. B 47, 558-561 (1993).

(6) Kresse, G. \& Hafner, J. Ab initio molecular-dynamics simulation of the liquid-metalamorphous semiconductor transition in germanium. Phys. Rev. B 49, 14251-14269 (1994).

(7) Kresse, G. \& Furthmüller, J. Efficiency of ab-initio total energy calculations for metals and semiconductors using a plane-wave basis set. Comput. Mater. Sci. 6, 15-50 (1996).

(8) Kresse, G. \& Furthmüller J. Efficient iterative schemes for ab initio total-energy calculations using a plane-wave basis set. Phys. Rev. B 54, 11169-11186 (1996).

(9) Hammer, B.; Hansen, L. B.; Nørskov, J. K. Improved adsorption energetics within density-functional theory using revised Perdew-Burke-Ernzerhof functionals Phys. Rev. B. 59, 7413-7421 (1999).

(10) Kresse, G. \& Joubert, D. From ultrasoft pseudopotentials to the projector augmentedwave method. Phys. Rev. B 59, 1758-1775 (1999). 
(11) Blöchl, P., Jepsen, O. \& Andersen, O. Improved tetrahedron method for Brillouin-zone integrations. Phys. Rev. B 49, 16223-16233 (1994).

(12) Wilson, J.A. \& Yoffe, A.D. The transition metal dichalcogenides discussion and interpretation of the observed optical, electrical and structural properties. Adv. Phys. 18, 193-335 (1969).

(13) Li, H.; Tsai, C.; Koh, A. L.; Cai, L.; Contryman, A. W.; Fragapane, A. H.; Zhao, J.; Han, H. S.; Manoharan, H. C.; Abild-Pedersen, F.; Nørskov, J. K.; Zheng, X. Activating and Optimizing $\mathrm{MoS}_{2}$ Basal Planes for Hydrogen Evolution through the Formation of Strained Sulphur Vacancies. Nat. Mater. 2015, 15, 48.

(14) Jaramillo, T. F.; Jørgensen, K. P.; Bonde, J.; Nielsen, J. H.; Horch, S.; Chorkendorff, I. Identification of Active Edge Sites for Electrochemical $\mathrm{H}_{2}$ Evolution from $\mathrm{MoS}_{2}$ Nanocatalysts. Science 2007, 317 (5834), 100-102. 
